\title{
Article
}

\section{The Proton-Sensing GPR4 Receptor Regulates Paracellular Gap Formation and Permeability of Vascular Endothelial Cells}

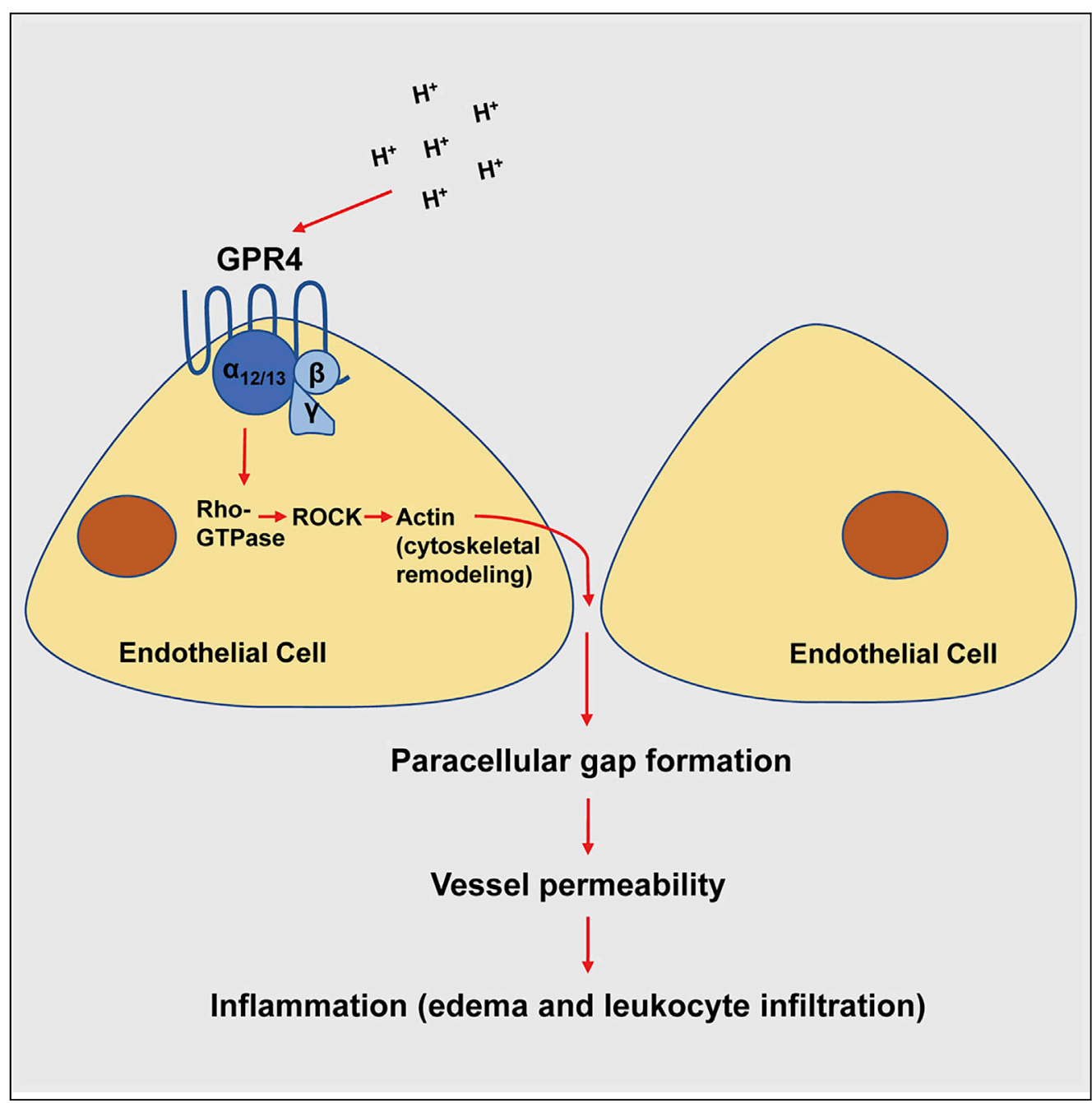

Elizabeth A.

Krewson, Edward

J. Sanderlin, Mona

A. Marie, Shayan

Nik Akhtar, Juraj

Velcicky, Pius

Loetscher, Li V.

Yang

yangl@ecu.edu

HIGHLIGHTS

Acidosis/GPR4 regulates endothelial paracellular gap formation and

permeability

GPR4 exacerbates

inflammation by

increasing tissue edema

and leukocyte infiltration

Pharmacological inhibition of GPR4

reduces inflammatory

responses 


\title{
The Proton-Sensing GPR4 Receptor Regulates Paracellular Gap Formation and Permeability of Vascular Endothelial Cells
}

\author{
Elizabeth A. Krewson, ${ }^{1}$ Edward J. Sanderlin, ${ }^{2}$ Mona A. Marie, ${ }^{2}$ Shayan Nik Akhtar, ${ }^{1}$ Juraj Velcicky, ${ }^{3}$ \\ Pius Loetscher, ${ }^{3}$ and Li V. Yang ${ }^{1,2,4, *}$
}

\begin{abstract}
SUMMARY
GPR4 is a pH-sensing G protein-coupled receptor highly expressed in vascular endothelial cells and can be activated by protons in the inflamed tissue microenvironment. Herein, we report that acidosisinduced GPR4 activation increases paracellular gap formation and permeability of vascular endothelial cells through the $\mathrm{G}_{\alpha 12 / 13} / \mathrm{Rh}$ o GTPase signaling pathway. Evaluation of GPR4 in the inflammatory response using the acute hindlimb ischemia-reperfusion mouse model revealed that GPR4 mediates tissue edema, inflammatory exudate formation, endothelial adhesion molecule expression, and leukocyte infiltration in the inflamed tissue. Genetic knockout and pharmacologic inhibition of GPR4 alleviate tissue inflammation. These results suggest GPR4 is a pro-inflammatory receptor and could be targeted for therapeutic intervention.
\end{abstract}

\section{INTRODUCTION}

The endothelium is a dynamic barrier that can mediate the transvascular movement of fluids and immune cells between the peripheral blood and interstitial tissues. During active inflammation, the production of numerous inflammatory mediators within the inflammatory loci can increase endothelial gap formation and vascular permeability, which facilitate leukocyte infiltration and exudate formation in the inflamed tissues (Edlow and Sheldon, 1971; Muller, 2003; Pate et al., 2010). Accumulating evidence suggests that microenvironmental factors, such as acidic $\mathrm{pH}$, can stimulate endothelial cell inflammation (Chen et al., 2011; Dong et al., 2013, 2017b; Okajima, 2013; Tobo et al., 2015). Inflammatory tissues are characteristically acidic, owing in part to hypoxia and increased glycolytic metabolism of cells and infiltrated leukocytes resulting in heightened proton production and accumulation. The acidic tissue microenvironment is associated with a wide range of inflammation-related disease states such as arthritis, inflammatory bowel disease, myocardial infarction, stroke, and limb ischemia. Previous reports note that local pH ranging from 6.0 to 7.0 is common in the microenvironments of inflamed tissues, solid tumors, and ischemic tissues (Huang and McNamara, 2004; Justus et al., 2013; Lardner, 2001; Siesjo et al., 1996). In ischemic disease, one study demonstrated that, within 50 min of coronary artery occlusion, local tissue extracellular $\mathrm{pH}$ decreased from 7.4 to 5.5 in domestic pigs (Hirche et al., 1980). Furthermore, in the tourniquet-induced rabbit limb ischemia model, local tissue $\mathrm{pH}$ decreased rapidly within $1 \mathrm{~h}$ and dropped from 7.30 to 6.36 during the 4-h course of limb ischemia (Hagberg, 1985). In summary, an acidic interstitial pH is an inflammatory microenvironmental factor in many pathological conditions and has been demonstrated to modulate tissue, blood vessel, and immune cell functions (Huang and McNamara, 2004; Justus et al., 2013; Lardner, 2001; Siesjo et al., 1996).

Cells can sense extracellular acidification through multiple molecular sensors such as acid-sensing ion channels (ASICs), transient receptor potential (TRP), and proton-sensing G protein-coupled receptors (GPCRs) (Holzer, 2009; Justus et al., 2013; Okajima, 2013; Sanderlin et al., 2015). GPR4 is a member of the proton-sensing GPCR family, which also includes GPR65 (TDAG8) and GPR68 (OGR1) (Justus et al., 2013; Ludwig et al., 2003; Okajima, 2013; Sanderlin et al., 2015; Yang et al., 2007). GPR4 is highly expressed in vascular endothelial cells (ECs) and has been shown to increase the expression of inflammatory cytokines, chemokines, adhesion molecules, and ER stress-related genes upon activation by acidic pH in ECs (Chen et al., 2011; Dong et al., 2013; Tobo et al., 2015). GPR4 has also been shown to potentiate inflammation in vivo. Recent studies found that the genetic deletion of GPR4 in mouse colitis models decreased the expression of endothelial adhesion molecules VCAM-1 and E-Selectin in the intestinal microvasculature,

${ }^{1}$ Department of Anatomy and Cell Biology, East Carolina University, Greenville, NC 27834, USA

${ }^{2}$ Department of Internal Medicine, Brody School of Medicine, East Carolina University, Greenville, NC 27834, USA

${ }^{3}$ Novartis Institutes for BioMedical Research, 4002 Basel, Switzerland

${ }^{4}$ Lead Contact

*Correspondence: yangl@ecu.edu

https://doi.org/10.1016/j.isci. 2020.100848 


\section{iScience}

which was associated with reduced mucosal leukocyte infiltration and intestinal inflammation (Sanderlin et al., 2017; Wang et al., 2018). Furthermore, GPR4 was shown to increase tissue injury in a renal ischemia-reperfusion mouse model (Dong et al., 2017a).

Our current study focuses on the acidosis/GPR4-mediated endothelial paracellular gap formation and vessel permeability in the inflammatory response. Using genetic and pharmacological approaches, we demonstrate that activation of GPR4 by acidosis induces endothelial paracellular gap formation and permeability through the $\mathrm{G} \alpha_{12 / 13}$ signaling pathway. Furthermore, we demonstrate that the genetic deletion and pharmacological inhibition of GPR4 decrease blood vessel permeability, tissue edema, and leukocyte infiltration in the acute hindlimb ischemia-reperfusion mouse model. Our data suggest that GPR4 has a proinflammatory role in the regulation of the inflammatory response.

\section{RESULTS}

\section{Acidosis Promotes Paracellular Gap Formation in Primary Endothelial Cells}

Tissue acidosis commonly exists in inflammatory microenvironments (Dong et al., 2014b; Justus et al., 2013; Lardner, 2001; Okajima, 2013; Sanderlin et al., 2015). However, the involvement of acidosis in endothelial cell (EC) gap formation is largely unknown. Four primary vascular ECs were cultured to a confluent monolayer and were treated with either physiological pH 7.4 or acidic $\mathrm{pH} 6.4$ for $5 \mathrm{~h}$ to assess acidosis-induced paracellular gap formation. Under physiological pH 7.4, all ECs maintained a cellular monolayer with no gap formation over the 5-h time course. However, under acidic $\mathrm{pH} 6.4$ the cellular monolayers were disrupted and paracellular gap formation was observed (Figure 1 A). The gap formation of EC monolayers was determined in human umbilical vein endothelial cells (HUVECs), human pulmonary artery ECs (HPAECs), human colon microvascular ECs (HMVECs-Colon), and human lung microvascular ECs (HMVECs-Lung) by calculating the total percent area of gaps in each field of view (Figures 1B-1E). ECs treated with acidic $\mathrm{pH} 6.4$ for $5 \mathrm{~h}$ developed approximately $4 \%-5 \%$ gap area relative to the total area (Figures 1B-1E). No gaps, however, could be detected within physiological $\mathrm{pH}$ conditions.

\section{Acidosis Stimulates Endothelial Paracellular Gap Formation through the Proton-Sensing GPR4 Receptor}

To determine the role of the $\mathrm{pH}$-sensing receptor GPR4 in acidosis-induced EC gap formation, we used genetic and pharmacological approaches to modulate GPR4 expression and activity, respectively. HUVECs were stably transduced with either control (HUVEC/vector), GPR4 overexpression (HUVEC/GPR4), or GPR4 signaling-defective mutant (HUVEC/GPR4 R115A) overexpression constructs. GPR4 knockdown was achieved with transduction of GPR4 shRNA (HUVEC/GPR4 shRNA) and compared with control shRNA (HUVEC/control shRNA). As previously described (Chen et al., 2011; Dong et al., 2013), real-time RT-PCR analysis showed that GPR4 mRNA expression was increased by $\sim 14$-fold in the overexpression HUVEC cells and decreased by $>90 \%$ in the shRNA knockdown cells (Figure S1). HUVECs were evaluated under physiological pH 7.4 or acidic $\mathrm{pH} 6.4$ for 5 h. Treatment with physiological pH 7.4 resulted in no observable gap formation. However, under acidic pH 6.4, HUVEC/vector cell monolayers developed $4 \%$ gap formation (Figure 2A). Overexpression of GPR4 significantly increased acidosis-induced gap formation by $\sim 2.5$ fold ( 10\%-11\%) under acidic conditions when compared with HUVEC/vector. Conversely, HUVEC/GPR4 R115A mutant decreased the percentage of gap area when compared with HUVEC/vector ( $1.8 \%$ versus $\sim 4 \%$, respectively). Furthermore, knockdown of GPR4 by shRNA decreased the percentage of gap area to $\sim 2.5 \%$ in acidic $\mathrm{pH} 6.4$ conditions when compared with HUVEC/control shRNA ( 4.5\%) (Figure $2 \mathrm{~B}$ ).

We next assessed the effects of a GPR4 inhibitor (EIDIP) on acidosis-induced paracellular gap formation in HUVEC/vector and HUVEC/GPR4 cells (Figures 2C and 2D). Pharmacological inhibition of GPR4 attenuated acidosis-induced gap formation. A dose-dependent decrease in the acidosis-induced gap development could be observed with increasing inhibitor concentrations during HUVEC/vector and HUVEC/GPR4 treatments when compared with vehicle (Figures $2 \mathrm{C}$ and 2D). The results indicate that acidosis-induced EC paracellular gap formation is dependent on GPR4 activation by acidic $\mathrm{pH}$.

\footnotetext{
GPR4-Mediated $\alpha_{12 / 13} /$ Rho GTPase Signaling Is Involved in Endothelial Paracellular Gap Formation in Response to Acidosis

Previous studies demonstrate that the $\mathrm{G} \alpha_{12 / 13} /$ Rho GTPase pathway can regulate cytoskeletal dynamics, endothelial gap formation, and endothelial permeability (Thennes and Mehta, 2012; van Buul and Hordijk,
} 
A

$0 \mathrm{hr}$
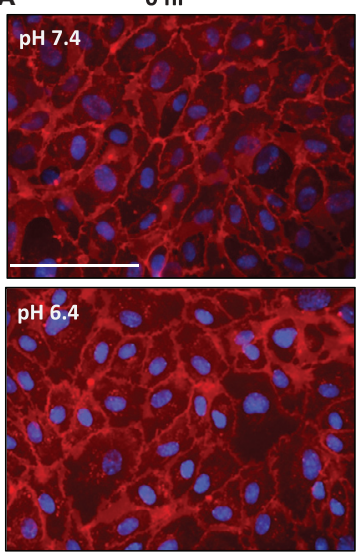

B

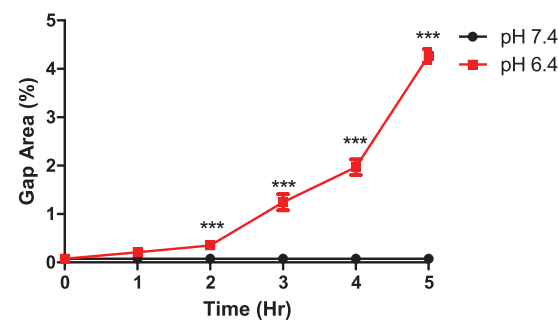

D

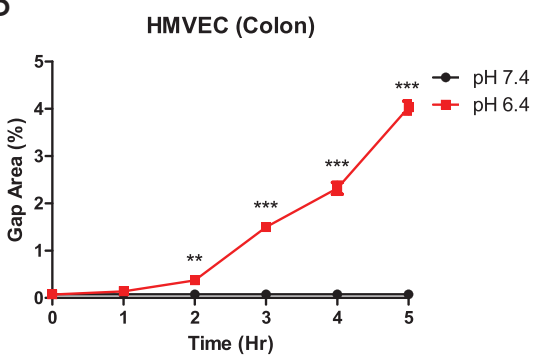

$3 \mathrm{hr}$
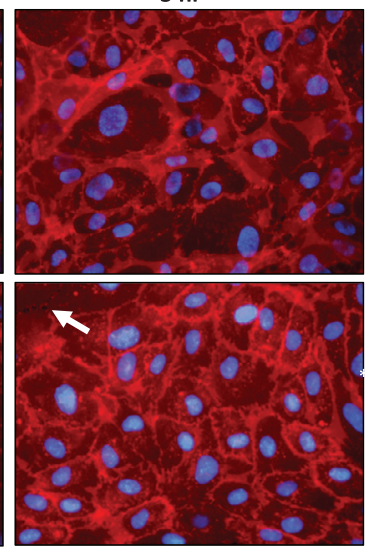

C
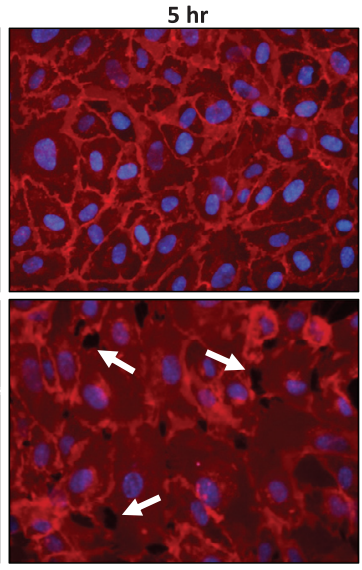

HPAEC

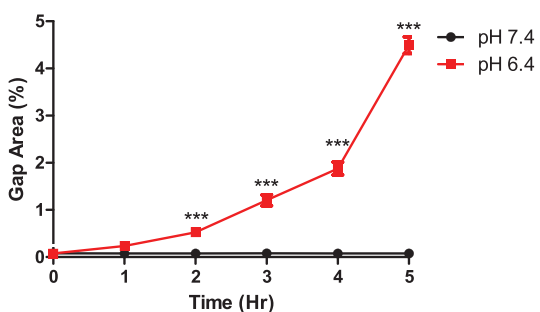

$\mathbf{E}$

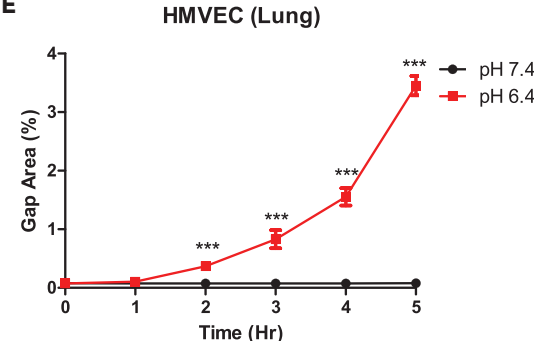

Figure 1. Acidosis Stimulates Paracellular Gap Formation in Primary Vascular Endothelial Cells (ECs) Plasma membrane staining and paracellular gap area quantitation of ECs treated for up to $5 \mathrm{~h}$ under physiological or acidic $\mathrm{pH}$. Acidosis increases EC gap formation when compared with physiological $\mathrm{pH}$ treatment conditions.

(A) Representative pictures of plasma membrane staining in human umbilical vein endothelial cells (HUVECs) at 0, 3, and $5 \mathrm{~h}$ treated under physiological or acidic $\mathrm{pH}$.

(B-E) Quantitative analysis of gap formation in (B) HUVECs, (C) human pulmonary artery endothelial cells (HPAECs), (D) human colon microvascular endothelial cells (HMVEC-Colon), and (E) human lung microvascular endothelial cells (HMVEC-Lung) over $5 \mathrm{~h}$. All experiments were performed in triplicate and are representative of four experiments. Data at each time point are presented as mean \pm SEM and analyzed for statistical significance between the $\mathrm{pH} 7.4 \mathrm{group}$ and the $\mathrm{pH} 6.4$ group using the unpaired t test where ${ }^{* *} p<0.01$ and ${ }^{* * *} p<0.001$. White arrows point to paracellular gaps. Scale bar, $100 \mu \mathrm{m}$.

2004). In line with these observations, GPR4 can couple to $G \alpha_{12 / 13}$ and Rho GTPase when expressed in cancer cell lines (Castellone et al., 2011; Justus and Yang, 2015). For this reason, we investigated the role of the GPR4/G $\alpha_{12 / 13} /$ Rho GTPase pathway in acidosis-induced EC gap formation. The p115 RGS G $\alpha_{12 / 13}$ inhibitory construct (Kozasa et al., 1998; Yang et al., 2005) was stably transduced into HUVEC/vector and HUVEC/GPR4 cells. We next performed the gap formation assay under physiological and acidic $\mathrm{pH}$ conditions for 5 h. HUVEC/p115 RGS cells treated with acidic $\mathrm{pH}$ had significantly reduced gap formation when compared with the vector control (Figures $3 \mathrm{~A}$ and $3 \mathrm{~B}$ ). Furthermore, thiazovivin and staurosporine, two chemical inhibitors for $\mathrm{G}_{12 / 13}$ downstream effectors Rho-associated kinase (ROCK) and myosin light-chain kinase (MLCK), respectively (Justus and Yang, 2015), were used in HUVEC/vector and HUVEC/GPR4 cells 
A
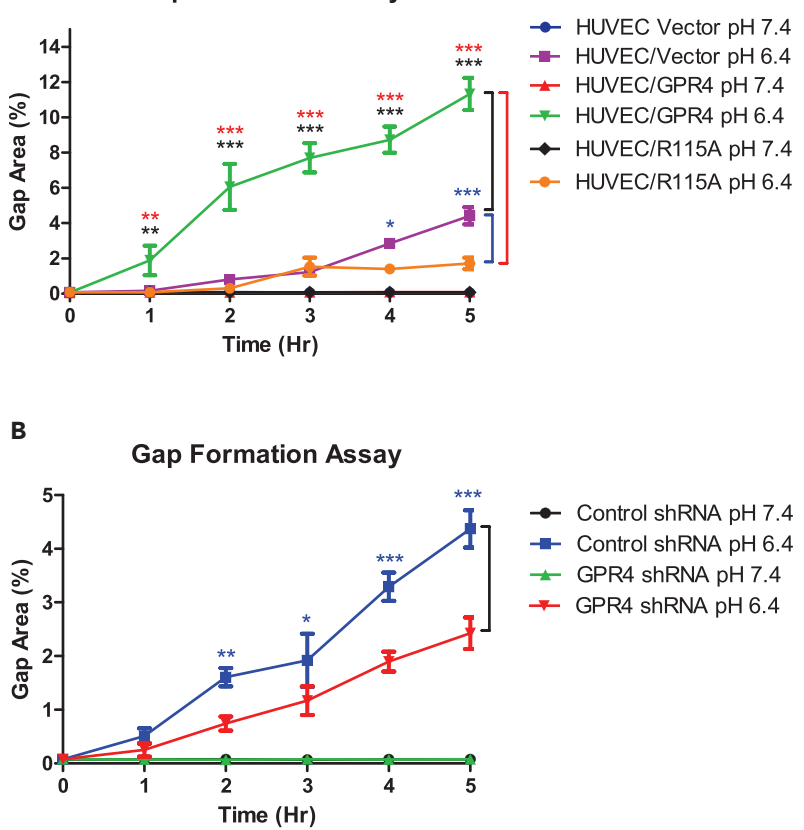

C Gap Formation Assay

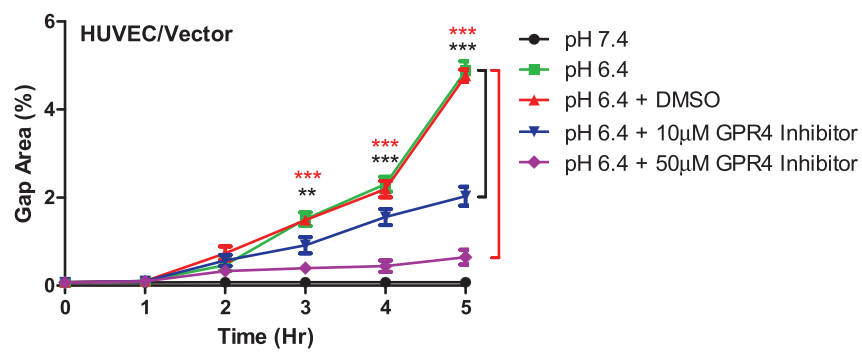

D

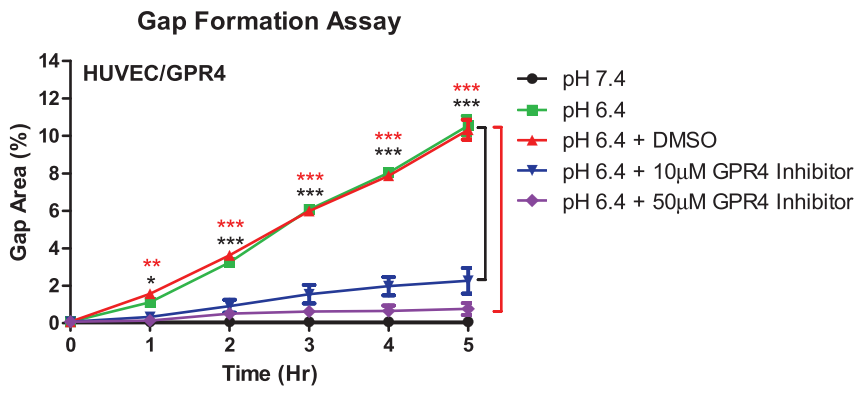

Figure 2. Activation of GPR4 by Acidosis Induces Paracellular Gap Formation in HUVECs

(A-D) Quantitative analysis of gap formation utilizing genetic and pharmacological approaches to modulate GPR4 expression or activity. (A) HUVEC/vector, HUVEC/GPR4, and HUVEC/R115A cells. (B) HUVEC/control shRNA and HUVEC/GPR4 shRNA cells. (C) HUVEC/vector and (D) HUVEC/GPR4 cells treated with GPR4 inhibitor or vehicle. All cells were treated with physiological pH 7.4 or acidic $\mathrm{pH} 6.4$ for $0,1,2,3,4$, and $5 \mathrm{~h}$ and the percent of gap area was quantified. DMSO was used as vehicle control. All experiments were performed in duplicate or triplicate and are representative of three experiments. Data at each time point are presented as mean \pm SEM and analyzed for statistical significance using the ANOVA where * $p<0.05,{ }^{\star *} p<0.01$, and ${ }^{\star \star *} p<0.001$. Black, blue, and red symbols indicate statistical analysis between groups indicated by bracket markers.

under acidic conditions. Thiazovivin significantly decreased gap formation percentage in HUVEC/vector and HUVEC/GPR4 cells when compared with the vehicle controls under acidic $\mathrm{pH}$. Staurosporine nearly abolished acidosis-induced gap formation in HUVEC/vector and HUVEC/GPR4 cells (Figures 3C and 3D). Collectively, the results suggest that acidosis-induced EC gap formation relies, at least in part, on the GPR4/G $\alpha_{12 / 13}$ pathway. Additionally, our results demonstrated that acidosis also induced F-actin stress fiber formation and decreased VE-cadherin expression at the site of paracellular gaps in ECs (Figures S2 and S3). To assess the role of actin cytoskeleton in acidosis/GPR4-mediated EC gap formation, HUVECs were treated with an actin cytoskeleton inhibitor cytochalasin D (CytoD). Cytochalasin D significantly reduced acidosis-induced gap formation in HUVEC/vector and HUVEC/GPR4 cells (Figures 3C and 3D). Moreover, p115 RGS, thiazovivin, staurosporine, and cytochalasin D substantially decreased F-actin stress fiber formation in HUVECs (Figure S4). Taken together, these results suggest that the G $\alpha_{12 / 13} /$ Rho/ROCK/ MLCK/actin cytoskeleton pathway is important for acidosis/GPR4-induced endothelial paracellular gap formation.

\section{Acidosis Increases Endothelial Cell Permeability through GPR4}

We next assessed if GPR4-dependent paracellular gap formation can functionally result in increased endothelial permeability. Endothelial permeability was assessed by the fluorescein isothiocyanate conjugateddextran (FITC-dextran) permeability assay whereby diffusion of FITC-dextran through the endothelial monolayer from the upper to lower chamber of the Transwell insert was assessed. Cells were treated with physiological $\mathrm{pH} 7.4$ or acidic $\mathrm{pH} 6.4$ for $5 \mathrm{~h}$ followed by the addition of FITC-dextran. Acidic pH treatment significantly increased FITC-dextran permeability in HUVEC/vector cells when compared with physiological $\mathrm{pH}$. Moreover, when GPR4 is overexpressed and treated with acidic $\mathrm{pH}$ there was a further increase in acidosis-induced FITC-dextran when compared with HUVEC/vector cells. When GPR4 expression is knocked down or signaling is defective (R115A mutant) (Chen et al., 2011), FITC-dextran permeability was significantly decreased compared with controls under acidic conditions (Figure 4). These results suggest that acidosis increases EC permeability through GPR4. 


\section{G $\alpha_{12 / 13}$ Inhibitory Construct}

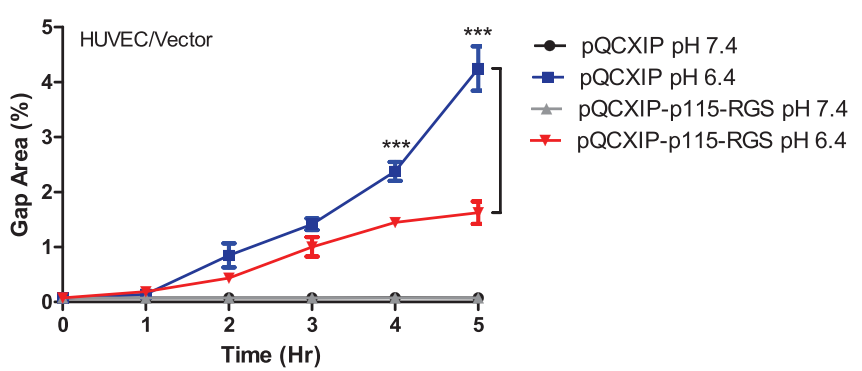

B

\section{G $\alpha_{12 / 13}$ Inhibitory Construct}

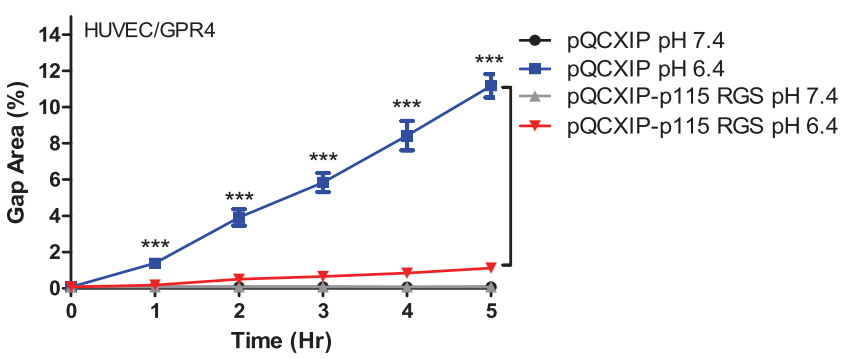

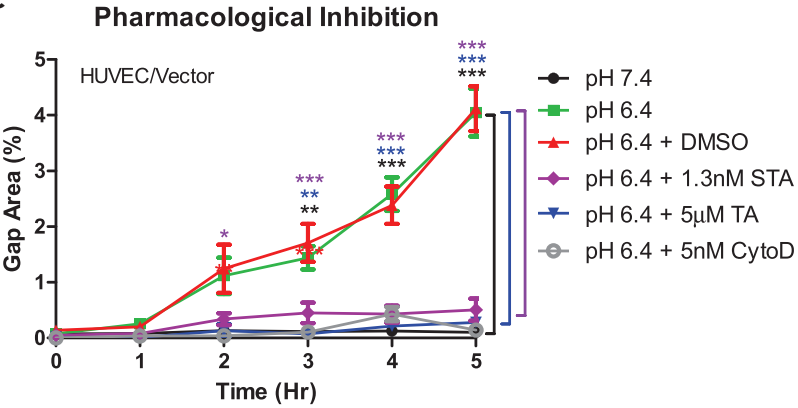

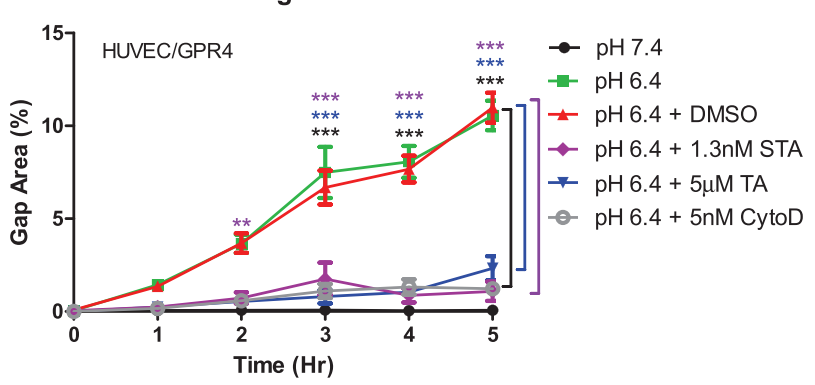

Figure 3. The $\mathrm{G}_{12 / 13} /$ Rho GTPase Pathway Is Involved in Acidosis/GPR4-Mediated Paracellular Gap Formation in HUVECs (A-D) Quantitative analysis of GPR4/G $\alpha_{12 / 13}$-mediated paracellular gap formation in HUVECs. GPR4-mediated paracellular gap formation is dependent, at least in part, on the $\mathrm{G} \alpha_{12 / 13} / \mathrm{Rho}$ GTPase/ROCK/MLCK/actin cytoskeleton pathway in response to acidic $\mathrm{pH}$. HUVECs were treated with various inhibitors, including p115-RGS (an inhibitory construct of $\mathrm{G}_{\alpha 12 / 13}$ ), thiazovivin (a Rho-associated kinase ROCK inhibitor), staurosporine (a myosin light-chain kinase MLCK inhibitor), and cytochalasin D (an actin cytoskeleton inhibitor). The G $\alpha_{12 / 13}$ p115-RGS inhibitory construct in (A) HUVEC/vector and (B) HUVEC/GPR4 cells. Pharmacological inhibition of the $\mathrm{G} \alpha_{12 / 13} /$ Rho GTPase/ROCK/MLCK/actin cytoskeleton pathway using $5 \mu M$ thiazovivin (TA), $1.3 \mathrm{nM}$ staurosporine (STA), or $5 \mathrm{nM}$ cytochalasin D (CytoD) in (C) HUVEC/vector or (D) HUVEC/GPR4 cells. HUVECs were treated with either physiological pH 7.4 or acidic pH 6.4 for up to $5 \mathrm{~h}$. All experiments were performed in duplicate and are representative of at least three experiments. Data at each time point are presented as mean \pm SEM and analyzed for statistical significance using ANOVA where ${ }^{\star} p<0.05,{ }^{* *} p<0.01$, and ${ }^{* * *} p<0.001$. Black, blue, and purple symbols indicate statistical analysis between groups indicated by bracket markers.

\section{Genetic Deletion of GPR4 Reduces Tissue Edema and Inflammation in the Acute Hindlimb} Ischemia-Reperfusion Mouse Model

Next, we assessed the functional role of GPR4 in a tourniquet cuff-based acute hindlimb ischemia-reperfusion mouse model (Bonheur et al., 2004). This mouse model can cause severe inflammation resulting in increased vessel permeability, tissue edema, and leukocyte infiltration in the affected tissue. The ischemia-reperfusion associated inflammation was induced in wild-type (WT) and GPR4 knockout (KO) mice. Consistent with previous report (Yang et al., 2007), the absence of GPR4 mRNA expression in KO mouse tissues was confirmed by RT-PCR (Figure S5). The sham and cuff limbs were measured for circumference differences between pre- and post-procedure measurements. GPR4 KO mice had less observable tissue edema in the tourniquet-affected limb following the ischemia and reperfusion event when compared with WT mice (Figure 5).

Inflammatory exudates in the interstitial space between the skin and the limb muscle/body peritoneum on the tourniquet subjected side were collected and measured followed by histology (Figure 6). GPR4 KO mice had reduced inflammatory exudate when compared with the WT mice ( $~ 6$ versus $\sim 65 \mathrm{mg}$, respectively) (Figures 6A, 6B, and 6I). Furthermore, histological analysis of the inflammatory exudates revealed that GPR4 KO mice had reduced leukocyte infiltrates when compared with WT (Figures 6D, 6F, and 6J). No red blood cells were observed in the exudate, suggesting that the exudate is due to increased vascular permeability but not vessel hemorrhaging (Figures 6A-6F). To further assess the GPR4-mediated vessel permeability, we performed immunohistochemistry for plasma protein immunoglobulin $\mathrm{G}$ (IgG) and detected IgG within inflammatory exudates (Figures $6 \mathrm{G}$ and $6 \mathrm{H}$ ), indicating an increased vascular permeability to plasma protein. Furthermore, less plasma IgG could be observed in the GPR4 KO cuff-affected 
Permeability Assay

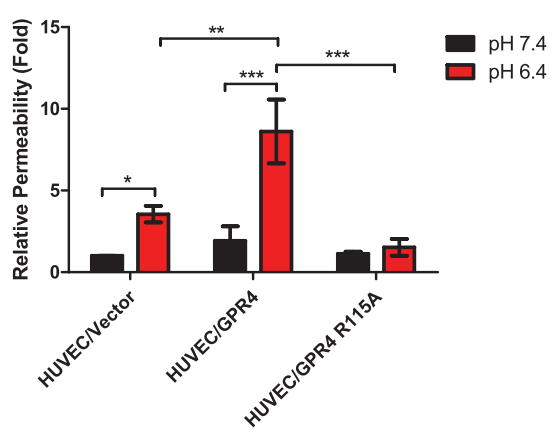

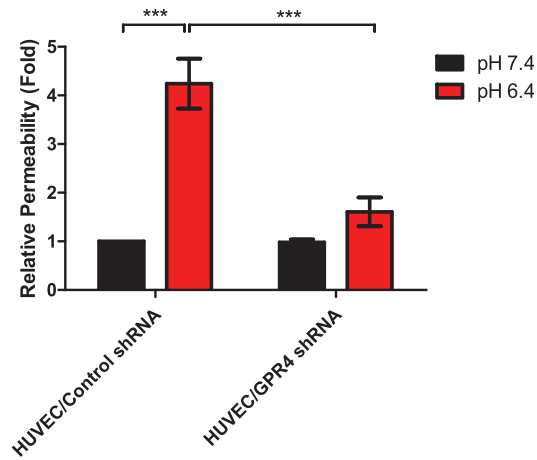

Figure 4. GPR4 Activation by Acidosis Increases Cellular Permeability in HUVECs

(A and B) Quantitative analysis of cellular permeability in vitro. GPR4 activation by acidic pH increases cellular permeability using the FITC-dextran cellular permeability assay with HUVECs. Relative fold increase of cellular permeability in (A) HUVEC/vector, HUVEC/GPR4, and HUVEC/R115A cells and (B) HUVEC/control shRNA and HUVEC/ GPR4 shRNA cells. Cells were treated for $5 \mathrm{~h}$ with either physiological pH 7.4 or acidic $\mathrm{pH}$ 6.4. All experiments were performed in duplicate and are representative of at least three experiments. Data are presented as mean \pm SEM and analyzed for statistical significance using ANOVA where ${ }^{*} p<0.05$, ${ }^{* *} p<0.01$, and ${ }^{* * *} p<0.001$.

tissues compared with WT cuff-affected tissues indicating reduced endothelial cell permeability in the KO (Figures $6 \mathrm{G}$ and $6 \mathrm{H}$ ). We also performed immunohistochemistry of CD31 (a pan-endothelial marker) to assess blood vessel density in the hindlimb dermis and hypodermis tissues. No significant difference in blood vessel density was observed between WT and GPR4 KO or between sham and cuff-affected dermis and hypodermis tissues within the 24-h ischemia and reperfusion (Figure S6).

To provide a molecular explanation for GPR4-mediated inflammation within vascular endothelial cells, we performed immunohistochemistry to examine the expression of VCAM-1 and E-selectin within the endothelium of the cuff and sham-affected dermis and hypodermis tissues. Overall, VCAM-1 and E-selectin protein expression was increased within the cuff-affected limb vasculature when compared with sham (Figure 7). Immunohistochemical analysis of VCAM-1 revealed expression on a variety of cell types, such as skeletal muscle, fibroblast, and vascular endothelial cells, which is consistent with the previous literature (Epperly et al., 2002; Ulyanova et al., 2005). Interestingly, we observed a decrease of VCAM-1 protein

A

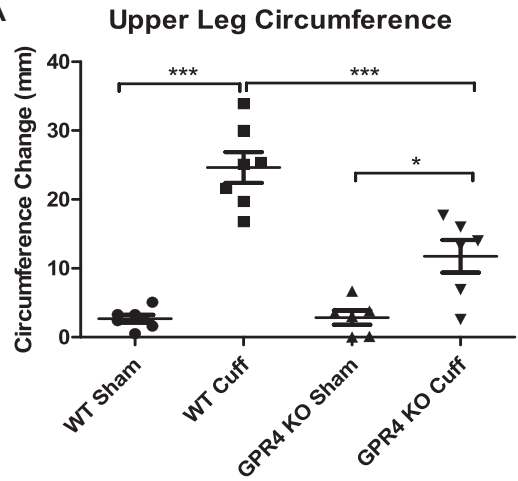

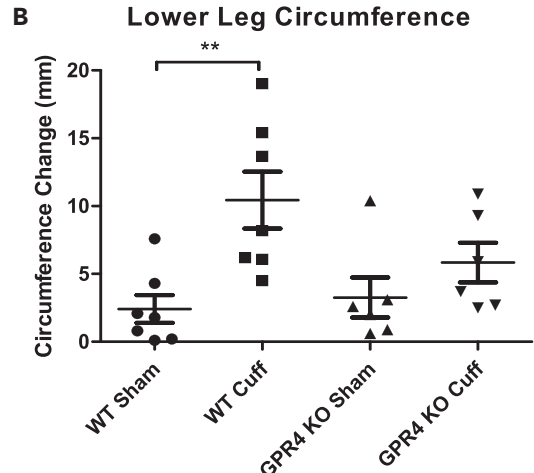

Figure 5. GPR4 Deficiency Reduces Tissue Edema in the Inflammatory Hindlimb Ischemia-Reperfusion (IR) Mouse Model

( $A$ and $B$ ) Upper and lower leg circumferences in wild-type (WT, N = 7) and GPR4 knockout (GPR4 KO, N=6) mice were measured before and after IR. The change of leg circumference was calculated (value after IR - value before IR) and used as an indicator for tissue edema. GPR4 deficiency reduces the change of upper and lower leg circumference when compared with WT mice in the IR mouse model. Quantitative analysis of (A) upper and (B) lower leg circumference changes. Data are presented as mean \pm SEM and analyzed for statistical significance using ANOVA where ${ }^{*} p<0.05$, ${ }^{* *} p<0.01$, and ${ }^{* * *} p<0.001$. 

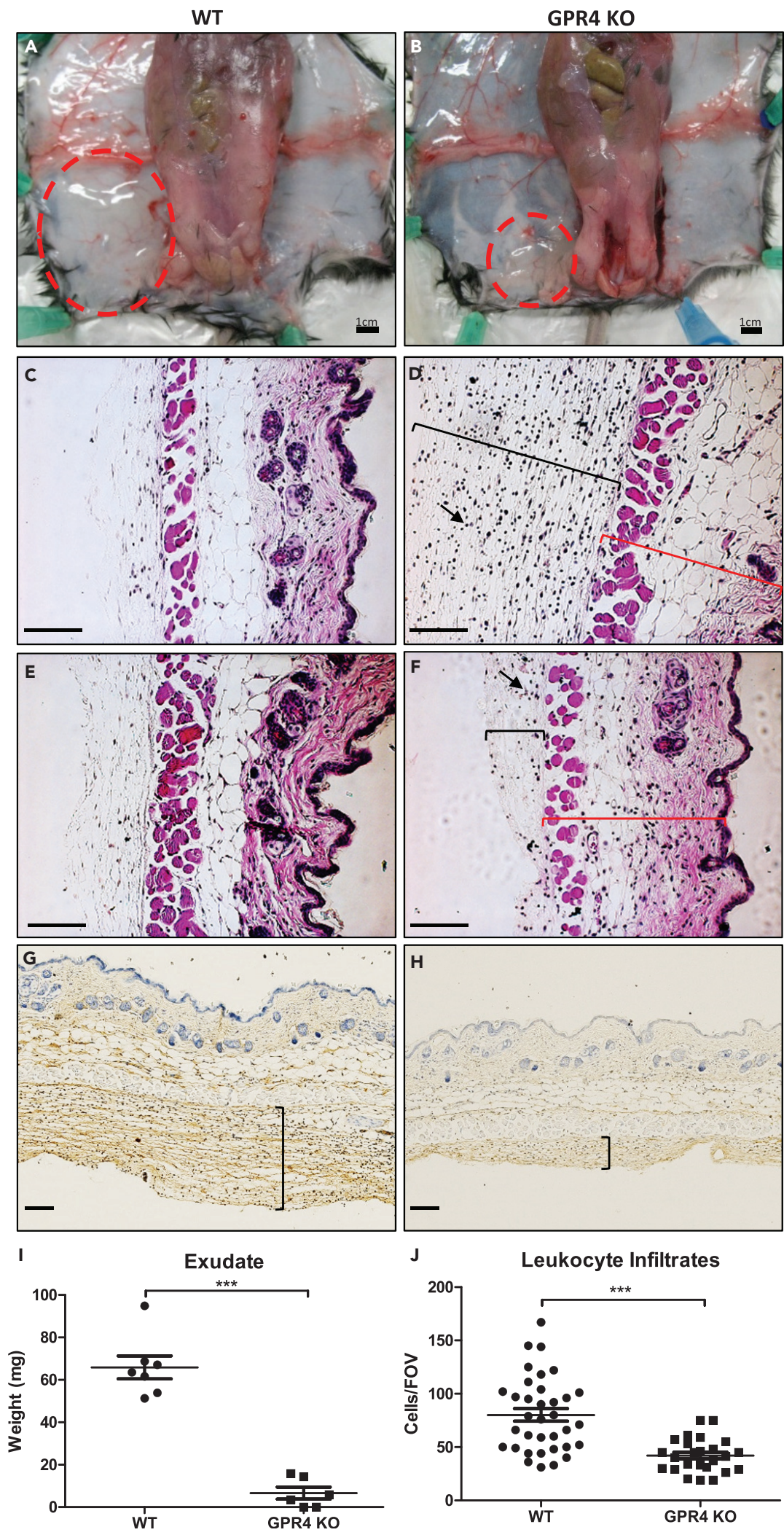
Figure 6. GPR4 Deficiency Reduces Inflammatory Exudate Production and Leukocyte Infiltration in the Ischemic Hindlimb Tissues

(A-J) Inflammatory exudate measurements and leukocyte infiltration quantification in WT and GPR4 KO mice following the ischemia-reperfusion of the hindlimb. Genetic deletion of GPR4 reduces inflammatory exudate production and leukocyte infiltration into the inflammatory exudate. Gross representative images of observable exudate formation in (A) WT and (B) GPR4 KO mice in the interstitial space between the skin and muscle of the ischemic hindlimb. Representative images of H\&E staining of (C) WT sham, (D) WT cuff, (E) GPR4 KO sham, and (F) GPR4 KO cuff inflammatory exudate sections. Black brackets indicate exudate distribution. Arrows indicate infiltrated leukocytes in the inflammatory exudate. Red brackets indicate skin tissues. Representative images of mouse plasma IgG protein in (G) WT cuff and (H) GPR4 KO cuff sections. IgG protein can be visualized as brown signal. The plasma protein IgG is used as a marker to indicate blood vessel permeability. Quantitative analysis of $(\mathrm{I})$ exudate weight and $(\mathrm{J})$ leukocyte infiltration from multiple fields of view (FOV). 10x and 20x microscope objectives used. Scale bar indicates $100 \mu \mathrm{m}$. Data analyzed from seven WT and six GPR4 $\mathrm{KO}$ mice. Data are presented as mean \pm SEM and analyzed for statistical significance using the unpaired two-tailed t test where ${ }^{* \star} p<0.001$

expression on vascular endothelial cells in GPR4 KO cuff-affected dermis and hypodermis of the limbs when compared with WT (Figures 7A-7D and 7I). E-selectin expression was observed on endothelial cells and fibroblasts as previously reported (Bajnok et al., 2017; Harashima et al., 2001; Vainer et al., 1998). Similar to VCAM-1 expression patterns, there is an observable decrease in endothelial E-selectin protein expression in GPR4 KO cuff-affected dermis and hypodermis of the limbs when compared with WT (Figures 7E-7H and $7 \mathrm{~J})$.

Pharmacological Inhibition of GPR4 Reduces Tissue Edema, Leukocyte Infiltration, and Vascular Permeability in the Hindlimb Ischemia-Reperfusion Mouse Model

To further assess the role of GPR4, we incorporated the use of a highly potent and selective GPR4 antagonist (referred to as GPR4 antagonist 13) (Velcicky et al., 2017) within the acute hindlimb ischemia-reperfusion mouse model. Our results demonstrated that the GPR4 antagonist 13 significantly decreased upper and lower limb tissue edema measured by the circumference of the leg compared with limbs of the vehicle control (Figure 8). In addition, there was significantly less leukocyte infiltration within the inflammatory exudate in the GPR4 antagonist 13-treated mice when compared with the vehicle-treated mice (Figures 9A, 9B, and 9F). Furthermore, the exudate weight was also decreased in GPR4 antagonist 13-treated mice when compared with vehicle (Figure 9E). Additionally, vascular permeability was assessed and there was a reduction in plasma lgG diffusion in the exudate by the treatment of the GPR4 antagonist 13 when compared with vehicle treatment (Figures 9C and 9D). GPR4 antagonist 13 treatment also reduced the level of an inflammatory marker, C-reactive protein (CRP), in the mouse serum (Figure S7). To further examine the role of GPR4 within ECs, we performed immunohistochemistry to analyze the expression of VCAM-1 and E-selectin within the endothelium of the cuff-affected dermis and hypodermis tissues. We observed that ECs in the GPR4 antagonist 13-treated mice had reduced VCAM-1 and E-selectin protein expression compared with the vehicle-treated mice (Figure 10). Taken together, these data suggest that GPR4 can mediate inflammation, vessel permeability, and leukocyte infiltration into inflamed tissues.

\section{DISCUSSION}

This study demonstrates that activation of GPR4 by acidic $\mathrm{pH}$ can stimulate endothelial inflammation through the mediation of paracellular gap formation and permeability by the $G \alpha_{12 / 13}$ pathway. We also evaluate the functional role of GPR4 in the inflammatory response within the hindlimb ischemia-reperfusion mouse model using GPR4-null mice and a selective GPR4 antagonist (Velcicky et al., 2017). These data suggest that both the genetic knockout and pharmacological inhibition of GPR4 reduce the inflammatory response in the ischemia-reperfusion mouse model. These results indicate GPR4 could be a valuable therapeutic target for the remediation of inflammatory disease states.

The inflamed tissue microenvironment has been characterized by a loss of $\mathrm{pH}$ homeostasis. Acidosis is a microenvironmental stress factor in which both stromal and infiltrated immune cells exist and that alters cellular function (Lardner, 2001; Okajima, 2013; Sanderlin et al., 2015). Interestingly, local acidosis has been described as a danger signal within ischemic and inflamed tissues that can promote inflammation (Rajamaki et al., 2013). However, the way by which cells sense the altered acidic microenvironment and subsequently alter the inflammatory response has only recently been investigated. Cells can employ distinct acid-sensing mechanisms such as TRPs, ASICs, and proton-sensing GPCRs (Holzer, 2009; Okajima, 2013; Sanderlin et al., 2015; Wemmie et al., 2006). Proton-sensing GPCRs are activated by acidic pH through 

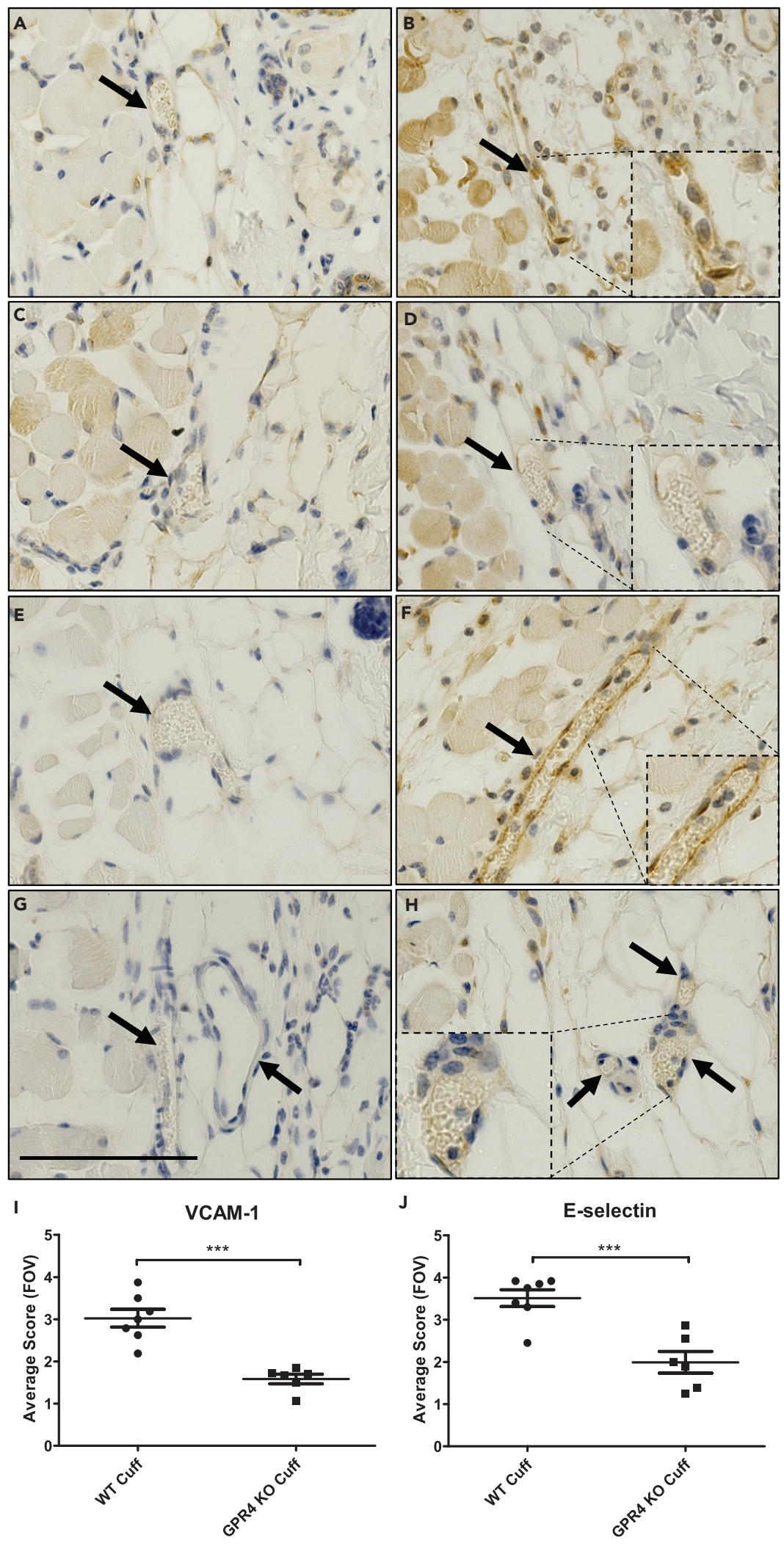


\section{iScience}

Figure 7. Immunohistochemistry of VCAM-1 and E-Selectin Protein Expression in the Loose Connective Dermal Tissue of WT and GPR4 KO Mice

(A-J) Immunohistochemical analysis of adhesion molecules VCAM-1 and E-selectin protein expression in vascular endothelial cells within mouse dermal tissue sections of sham and cuff affected hindlimbs. GPR4-deficient mice had reduced expression of VCAM-1 and E-selectin in vascular endothelial cells in cuff-affected tissues when compared with WT. Representative pictures of VCAM-1 signal in (A) WT sham, (B) WT cuff, (C) GPR4 KO sham, and (D) GPR4 KO cuff tissues. Representative pictures of E-selectin expression can be visualized in (E) WT sham, (F) WT cuff, (G) GPR4 KO sham, and (H) GPR4 KO cuff tissues. Quantitative scoring analysis of (I) VCAM-1 and (J) E-selectin protein expression in vascular endothelial cells in WT and GPR4 KO cuff-affected dermal tissues. Pictures taken with 63x objective. Scale bar indicates $100 \mu \mathrm{m}$. Arrows indicate blood vessels. Data are presented as mean \pm SEM and were analyzed for statistical significance using the unpaired two-tailed t test where ${ }^{* * *} p<0.001$.

the protonation of several histidine residues on the extracellular domains of the receptors, which can induce conformational changes in the GPCRs for subsequent $G$ protein activation and downstream signaling (Liu et al., 2010; Ludwig et al., 2003). The family of proton-sensing GPCRs include GPR4, GPR65 (TDAG8), and GPR68 (OGR1). GPR65 and GPR68 are predominately expressed in immune cells, whereas GPR4 is expressed in endothelial cells (Dong et al., 2014b; Justus et al., 2013; Okajima, 2013; Sanderlin et al., 2015). This family of receptors has only recently been implicated in the regulation of postischemic cellular responses and inflammation (Dong et al., 2014a; Ma et al., 2017; Matsuzaki et al., 2011; Russell et al., 2012; Sanderlin et al., 2015).

For example, GPR68 has been suggested to have a cardioprotective function by establishing a cellular protective border zone around the infarcted tissues (Russell et al., 2012). GPR65 expression was increased in rat brains and inhibited neuronal apoptosis and post-ischemic inflammatory responses following the middle cerebral artery occlusion and reperfusion event (Ma et al., 2017). Studies investigating the role of GPR4 under ischemic conditions are predominately focused on the kidney (Dong et al., 2014a). A recent study demonstrated that GPR4 deficiency improved renal ischemia-reperfusion injury clinical parameters such as survival rate, serum creatinine, and blood urea nitrogen levels. Furthermore, genetic deficiency of GPR4 deterred apoptosis by suppressing CHOP expression in the kidney (Dong et al., 2017a). This study is consistent with our previous report demonstrating CHOP expression is dependent on GPR4 activation in HUVECs (Dong et al., 2013, 2017b). However, these previous studies did not investigate the role of GPR4 in post-ischemic vessel permeability or inflammation.

Our current study reveals a functional role for GPR4 in governing endothelial paracellular gap formation and permeability in response to acidic stress through the $G \alpha_{12 / 13}$ pathway. Using both genetic and pharmacological approaches, we demonstrated that acidosis-induced GPR4 activation significantly increases the paracellular gap formation and permeability when compared with physiological $\mathrm{pH}$ in vitro. The acidosis/GPR4-induced HUVEC paracellular gap formation is mediated through the $\mathrm{G} \alpha_{12 / 13} /$ Rho GTPase/ROCK/actin cytoskeleton pathway. It has been previously reported that activation of GPR4 by acidosis can stimulate several downstream $G$ protein pathways including $G_{12 / 13}, G_{s}$, and $G_{q}$ (Liu et al., 2010; Ludwig et al., 2003). Future study is needed to elucidate the role of the complex signaling pathways of GPR4 in paracellular gap formation and other biological processes. Several potential mechanisms should be considered such as biased GPCR signaling and one G protein signaling pathway interacting with/overpowering another G protein signaling pathway. Other potential mechanisms, such as G protein-independent pathways and indirect effects due to cytokine/chemokine generation, should also be considered. Furthermore, a previous study reported that GPR4 can reduce endothelial cell barrier function via lysophosphatidylcholine (LPC) (Qiao et al., 2006). However, the LPC-GPR4 ligand binding interaction was unable to be confirmed (Zhu et al., 2001). Instead, GPR4 has been shown to function as a pH sensor (Ludwig et al., 2003; Yang et al., 2007). Altered endothelial cell permeability can lead to detrimental complications, such as tissue edema. Many diseases related to ischemia-induced inflammation are associated with increased vascular permeability such as stroke, myocardial infarction, sepsis, and cancer (Kumar et al., 2009). Our study suggests that GPR4 mediates endothelial paracellular gap formation and permeability in response to acidotic stress within the acidic inflamed microenvironment. For this reason, we further investigated the functional role of GPR4 in mediating acute inflammation using the hindlimb ischemia-reperfusion mouse model.

To investigate GPR4 in the ischemia-reperfusion model, we used the GPR4 KO mice for comparison with WT mice. Our data demonstrated that GPR4 KO mice had reduced parameters of acute inflammation 

Upper Leg Circumference

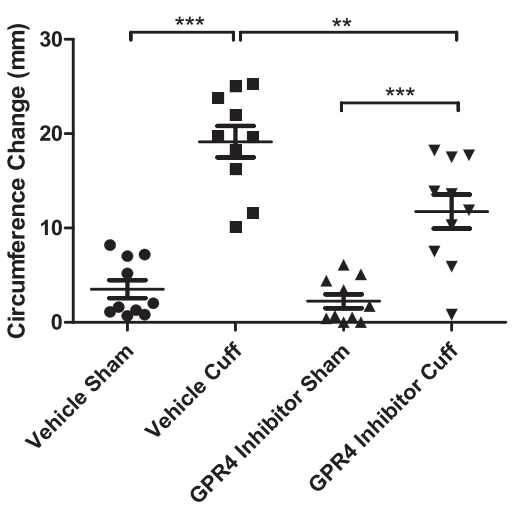

Lower Leg Circumference

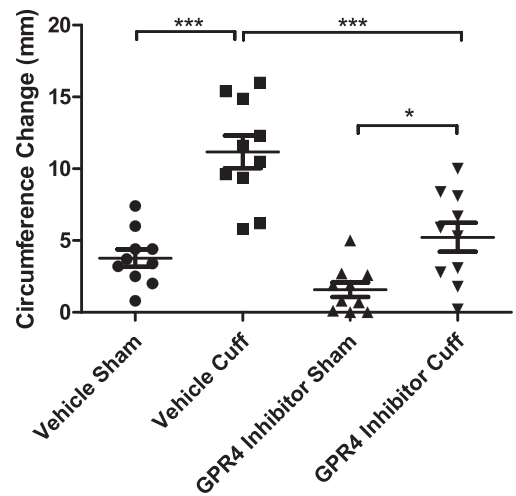

Figure 8. Pharmacological Inhibition of GPR4 Reduces Tissue Edema in the Inflammatory Hindlimb IschemiaReperfusion (IR) Mouse Model

( $A$ and B) Upper and lower leg circumference changes following IR in wild-type (WT) mice treated with either vehicle or GPR4 antagonist 13. GPR4 antagonist 13 reduces the changes of upper and lower leg circumference when compared with vehicle control in the IR mouse model. Quantitative analysis of (A) upper and (B) lower leg circumference changes, respectively. $\mathrm{N}=10$ vehicle- and $\mathrm{N}=10 \mathrm{GPR} 4$ antagonist 13-treated mice. Data are presented as mean $\pm \mathrm{SEM}$ and were analyzed for statistical significance using the ANOVA where ${ }^{*} p<0.05$, ${ }^{* *} p<0.01$, and ${ }^{* * *} p<0.001$.

such as tissue edema, inflammatory exudate formation, leukocyte infiltration, and EC adhesion molecule expression (VCAM-1 and E-selectin). These results suggest GPR4 mediates ischemia/reperfusion-induced inflammation most likely through vascular inflammatory programs such as increased vessel permeability and adhesion molecule expression. We and others have previously demonstrated that GPR4 regulates endothelial cell inflammation and ER stress responses (Chen et al., 2011; Dong et al., 2013, 2017b; Tobo et al., 2015). Activation of GPR4 in human endothelial cells by acidic pH increased numerous inflammatory cytokines, chemokines, cellular adhesion molecules, and ER stress-related genes (Dong et al., 2013, 2017b). Additionally, activation of GPR4 in HUVECs functionally mediated EC-leukocyte interactions, which are necessary for the leukocyte extravasation process in the inflammatory response (Chen et al., 2011; Dong et al., 2013). The role of GPR4 in the regulation of inflammation has recently been evaluated in vivo using the dextran sulfate sodium (DSS)-induced colitis mouse model (Sanderlin et al., 2017; Wang et al., 2018). GPR4 KO mice were protected from intestinal inflammation and had reduced adhesion molecule expression in intestinal microvascular endothelial cells when compared with WT mice (Sanderlin et al., 2017). These data suggest GPR4 potentiates inflammation likely through increased vascular endothelial cell inflammatory responses and are consistent with observations made in this current study and implicate GPR4 as a potential therapeutic target for the remediation of acute and chronic inflammation. It should also be noted that the GPR4 KO mice used in this study and other previous studies are global knockout with GPR4 deficiency in all tissue types (Dong et al., 2017a; Ludwig et al., 2003; Sanderlin et al., 2017; Wang et al., 2018; Yang et al., 2007). Although GPR4 is predominantly expressed in vascular endothelial cells, global knockout of GPR4 in other cell types might also have biological effects. Future research to generate endothelium-specific knockout mice will help further define the role of GPR4 in inflammatory responses and vascular biology.

Previous reports have indicated a group of imidazopyridine derivatives, found to selectively inhibit GPR4, can reduce both endothelial cell inflammation in vitro and tissue inflammation in vivo (Dong et al., 2013, 2017b; Fukuda et al., 2016; Miltz et al., 2017; Tobo et al., 2015). We previously demonstrated that GPR4 inhibitors can inhibit GPR4 activation in HUVECs following acidotic stimulation in vitro, which resulted in reduced expression of GPR4-mediated proinflammatory cytokines, chemokines, and cellular adhesion molecules (Dong et al., 2017b). Previous in vivo studies evaluated GPR4 inhibitors in myocardial infarction, arthritis, nociception, and angiogenesis mouse models and demonstrated that GPR4 inhibition reduced the disease severity when compared with vehicle control (Fukuda et al., 2016; Miltz et al., 2017). Recently, GPR4 antagonist 13, a pyrazolopyrimidine derivative was developed by Novartis Pharmaceuticals as the next generation of GPR4 inhibitors and found to be more selective for GPR4 inhibition and orally active (Velcicky et al., 2017). GPR4 antagonist 13 was also tested against other pH-sensing GPCRs, the H3 

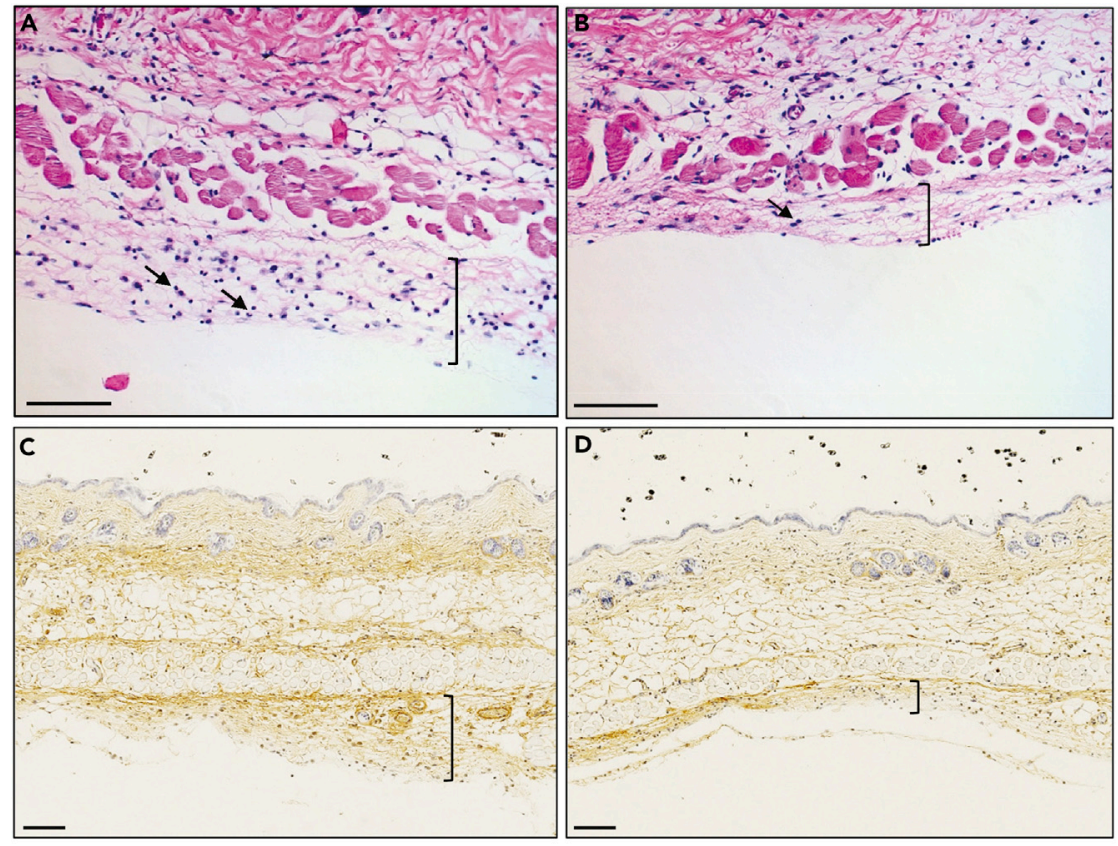

E

Exudate

$\mathbf{F}$

Leukocyte Infiltrates
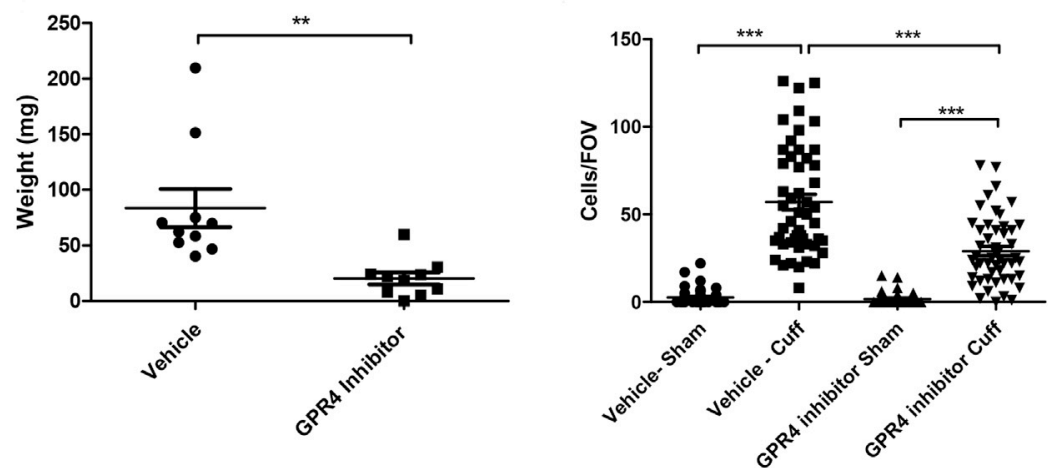

Figure 9. GPR4 Antagonist 13 Reduces Inflammatory Exudate Production and Leukocyte Infiltration in the Ischemic Hindlimb Tissues

(A-F) Inflammatory exudate measurements and leukocyte infiltration quantification in WT mice provided with vehicle or GPR4 antagonist 13 in the hindlimb ischemia-reperfusion mouse model. GPR4 antagonist 13 reduces inflammatory exudate formation and leukocyte infiltration in the exudate. Representative images of H\&E staining of (A) WT vehicle cuff and (B) WT GPR4 antagonist 13 cuff skin tissue sections. Immunohistochemical staining of mouse plasma IgG protein in (C) WT vehicle cuff and (D) WT GPR4 antagonist 13 cuff inflammatory exudate sections. IgG protein can be visualized as brown signal. (E) Total exudate formation and (F) leukocyte infiltration from multiple fields of view (FOVs) when compared with vehicle control following ischemia-reperfusion. Black brackets indicate exudate distribution. Arrows indicate infiltrated leukocytes. 10x and 20x microscope objectives used. Scale bars indicate $100 \mu \mathrm{m}$. Data analyzed from 10 vehicleand 10 GPR4 inhibitor-treated mice. Data are presented as mean \pm SEM and were analyzed for statistical significance using the unpaired two-tailed $t$ test or ANOVA where ${ }^{\star *} p<0.01$ and ${ }^{* \star} p<0.001$.

receptor, and hERG channel and demonstrated high selectivity for GPR4. The in vivo pharmacokinetics were also evaluated for GPR4 antagonist 13 and found to have good profiles of oral delivery and clearance. GPR4 antagonist 13 was found to effectively reduce arthritic inflammation, hyperalgesia, angiogenesis, and colitis (Sanderlin et al., 2019; Velcicky et al., 2017).

We sought to evaluate the anti-inflammatory effects of GPR4 antagonist 13 within the hindlimb ischemiareperfusion mouse model. Notably, we observed similar effects of the pharmacological inhibition of GPR4 with GPR4 antagonist 13 when compared with the genetic knockout of GPR4. Our results demonstrated 


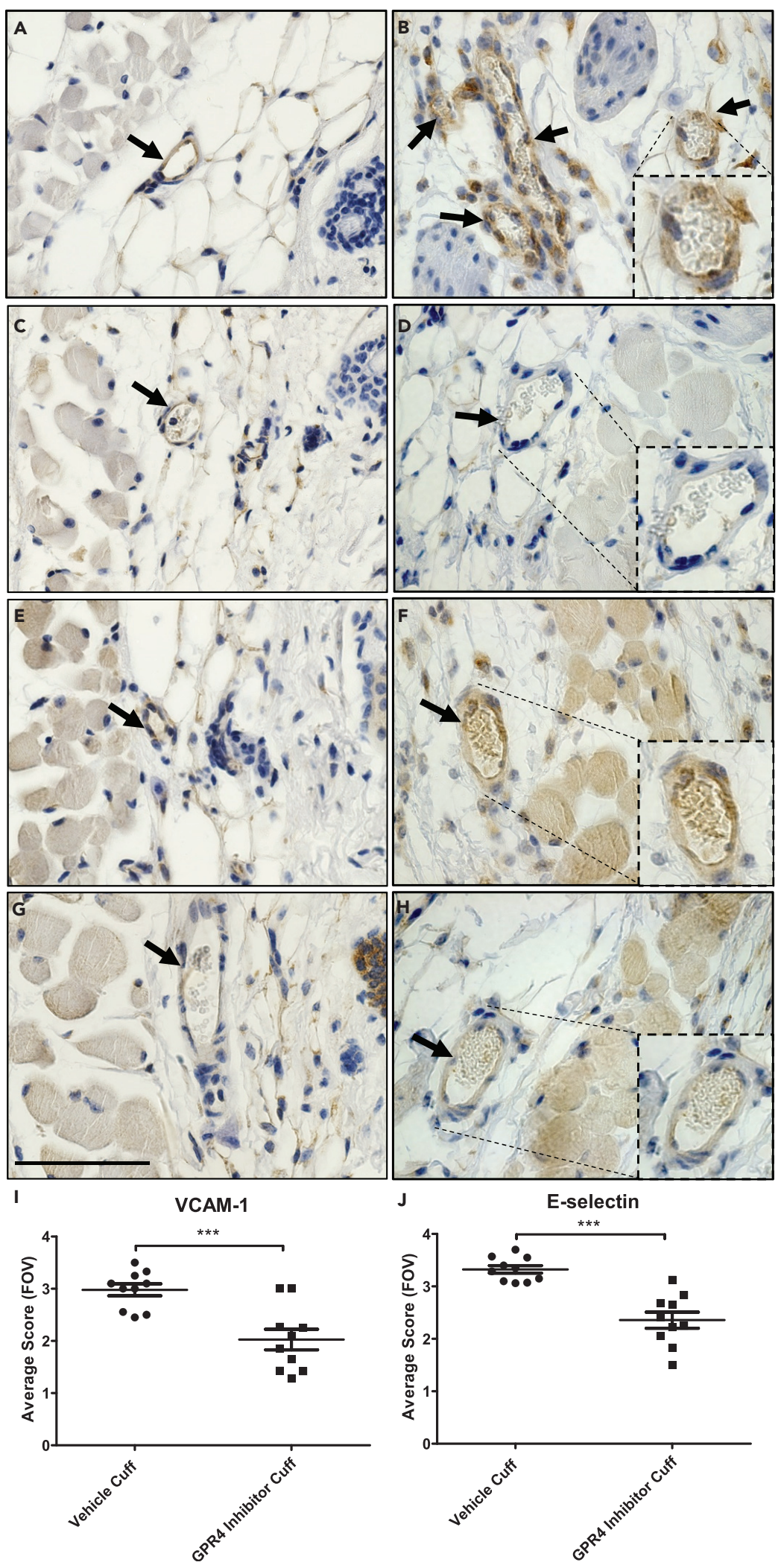


Figure 10. Immunohistochemistry of VCAM-1 and E-Selectin Protein Expression in the Loose Connective Dermal Tissue of Wild-Type Mice Treated with GPR4 Antagonist 13 or Vehicle

(A-J) Immunohistochemical analysis of adhesion molecules VCAM-1 and E-selectin protein expression in vascular endothelial cells within mouse dermal tissue sections of sham and cuff-affected hindlimbs. WT mice treated with GPR4 antagonist 13 had reduced expression of VCAM-1 and E-selectin in vascular endothelial cells in cuff-affected tissues when compared with vehicle control. Representative pictures of VCAM-1 signal in (A) WT vehicle sham, (B) WT vehicle cuff, (C) WT GPR4 antagonist 13 sham, and (D) WT GPR4 antagonist 13 cuff dermal connective tissues. Representative pictures of E-selectin expression can be visualized in (E) WT vehicle sham, (F) WT vehicle cuff, (G) WT GPR4 antagonist 13 sham, and (H) WT GPR4 antagonist 13 cuff dermal tissues. Quantitative scoring analysis of (I) VCAM-1 and (J) E-selectin protein expression in vascular endothelial cells in WT vehicle and WT GPR4 antagonist 13 cuff-affected dermal tissues. Pictures taken with 63x microscope objective. Scale bar indicates $100 \mu \mathrm{m}$. Arrows indicate blood vessels. Data are presented as mean \pm SEM and were analyzed for statistical significance using the unpaired two-tailed t test where ${ }^{\star \star *} p<0.001$.

that GPR4 exacerbated post-ischemia/reperfusion tissue inflammation. GPR4 antagonist 13 administration resulted in a decrease in gross edema clinical parameters, inflammatory exudate formation, and leukocyte infiltration. Moreover, GPR4 antagonist 13 treatment reduced endothelial permeability as evidenced by a decrease in plasma IgG protein leakiness when compared with vehicle control. Proinflammatory modulators such as endothelial adhesion molecules (VCAM-1 and E-selectin) were also decreased by GPR4 antagonist 13 compared with vehicle.

Taken together, this study demonstrates that GPR4 activation by acidosis can induce endothelial paracellular gap formation and permeability in vitro. Furthermore, evaluation of the contribution of GPR4 in the hindlimb post-ischemia/reperfusion inflammatory response supports the role for GPR4 in vascular permeability and inflammation. Genetic knockout and pharmacological inhibition of GPR4 in vivo can decrease leukocyte infiltration and the expression of endothelial adhesion molecules VCAM-1 and E-selectin and can reduce vascular permeability as evidenced by attenuated plasma lgG leakiness into the subcutaneous connective tissues and exudate formation. The results suggest that inhibition of GPR4 can be exploited as a potential approach to alleviate inflammation and tissue edema.

\section{Limitations of the Study}

This study focuses on the role of the proton-sensing receptor GPR4 in acidosis-mediated endothelial paracellular gap formation, permeability, and inflammatory response. In addition to the proton-sensing GPCRs, there are other types of acid sensors such as ASICs and TRPs (Holzer, 2009; Okajima, 2013; Sanderlin et al., 2015; Wemmie et al., 2006). It remains to be determined whether there are functional interactions between the proton-sensing GPCRs and other acid sensors in endothelial cell biology. Additionally, although this study and other preclinical studies demonstrate that the GPR4 antagonists exhibit anti-inflammatory, antinociceptive, and tissue-protective effects (Dong et al., 2013, 2017b; Fukuda et al., 2016; Miltz et al., 2017; Sanderlin et al., 2019; Tobo et al., 2015; Velcicky et al., 2017), the potential therapeutic effects of GPR4 antagonists in human patients remain to be evaluated.

\section{METHODS}

All methods can be found in the accompanying Transparent Methods supplemental file.

\section{SUPPLEMENTAL INFORMATION}

Supplemental Information can be found online at https://doi.org/10.1016/j.isci.2020.100848.

\section{ACKNOWLEDGMENTS}

This study was supported in part by research grants from the National Institutes of Health (R15DK109484, to L.V.Y.) and the American Heart Association (11SDG5390021, to L.V.Y.). We thank Nancy Leffler, Lixue Dong, Joani Oswald, and Comparative Medicine staff, Drs. Yan-Hua Chen, Warren Knudson, David Tulis, Karen Oppelt, and Kvin Lertpiriyapong for helpful discussion and technical assistance. We also thank the Novartis Institutes for BioMedical Research for providing the GPR4 antagonist 13 and Dr. Owen Witte for the GPR4 knockout mice.

\section{AUTHOR CONTRIBUTIONS}

E.A.K. and L.V.Y. designed the experiments; E.A.K., E.J.S., M.A.M., and S.N.A. performed the experiments; E.A.K., E.J.S., M.A.M., S.N.A., and L.V.Y. analyzed the data; L.V.Y. supervised the research; J.V. and P.L. 
provided the GPR4 antagonist 13 and instructions on how to administer the compound in mice; E.A.K., E.J.S., and L.V.Y. wrote the manuscript; all authors reviewed the manuscript.

\section{DECLARATION OF INTERESTS}

L.V.Y. is the inventor on a U.S. patent (US8207139B2) entitled "Function of GPR4 in vascular inflammatory response to acidosis and related methods." J.V. and P.L. are inventors on the patents related to the GPR4 antagonists used in this study, including US8748435B2 (US20120252778A1) entitled "Pyrazolo pyrimidine derivatives" and US20150216867A1 (WO2014049514A1) entitled "Compounds for use in gastric complication." J.V. and P.L. are employees of the Novartis Institutes for BioMedical Research. All other authors declare no competing interests.

Received: June 15, 2019

Revised: November 27, 2019

Accepted: January 13, 2020

Published: February 21, 2020

\section{REFERENCES}

Bajnok, A., Ivanova, M., Rigo, J., Jr., and Toldi, G. (2017). The distribution of activation markers and selectins on peripheral T lymphocytes in preeclampsia. Mediators Inflamm. 2017, 8045161.

Bonheur, J.A., Albadawi, H., Patton, G.M., and Watkins, M.T. (2004). A noninvasive murine model of hind limb ischemia-reperfusion injury. J. Surg. Res. 116, 55-63.

Castellone, R.D., Leffler, N.R., Dong, L., and Yang, L.V. (2011). Inhibition of tumor cell migration and metastasis by the proton-sensing GPR4 receptor. Cancer Lett. 312, 197-208.

Chen, A., Dong, L., Leffler, N.R., Asch, A.S., Witte, O.N., and Yang, L.V. (2011). Activation of GPR4 by acidosis increases endothelial cell adhesion through the cAMP/Epac pathway. PLoS One 6, e27586.

Dong, L., Krewson, E.A., and Yang, L.V. (2017b) Acidosis activates endoplasmic reticulum stress pathways through GPR4 in human vascular endothelial cells. Int. J. Mol. Sci. 18, 278.

Dong, L., Li, Z., Leffler, N.R., Asch, A.S., Chi, J.T., and Yang, L.V. (2013). Acidosis activation of the proton-sensing GPR4 receptor stimulates vascular endothelial cell inflammatory responses revealed by transcriptome analysis. PLoS One 8, e61991.

Dong, B., Zhou, H., Han, C., Yao, J., Xu, L., Zhang, M., Fu, Y., and Xia, Q. (2014a). Ischemia/ reperfusion-induced $\mathrm{CHOP}$ expression promotes apoptosis and impairs renal function recovery: the role of acidosis and GPR4. PLoS One 9, e110944.

Dong, L., Li, Z., and Yang, L.V. (2014b). Function and signaling of the $\mathrm{pH}$-sensing $\mathrm{G}$ protein-

coupled receptors in physiology and diseases. In Molecular Genetics of Dysregulated $\mathrm{pH}$

Homeostasis, J.T. Chi, ed. (Springer), pp. 45-65.

Dong, B., Zhang, X., Fan, Y., and Cao, S. (2017a). GPR4 knockout improves renal ischemiareperfusion injury and inhibits apoptosis via suppressing the expression of CHOP. Biochem. J. 474, 4065-4074.
Edlow, D.W., and Sheldon, W.H. (1971). The pH of inflammatory exudates. Proc. Soc. Exp. Biol. Med. 137, 1328-1332.

Epperly, M.W., Sikora, C.A., DeFilippi, S.J., Gretton, J.E., Bar-Sagi, D., Archer, H., Carlos, T. Guo, H., and Greenberger, J.S. (2002). Pulmonary irradiation-induced expression of VCAM-I and ICAM-I is decreased by manganese superoxide dismutase-plasmid/liposome (MnSOD-PL) gene therapy. Biol. Blood Marrow Transplant. 8, 175-187.

Fukuda, H., Ito, S., Watari, K., Mogi, C., Arisawa, M. Okajima, F. Kurose, H., and Shuto, S. (2016) Identification of a potent and selective GPR4 antagonist as a drug lead for the treatment of myocardial infarction. ACS Med. Chem. Lett. 7, 493-497.

Hagberg, H. (1985). Intracellular pH during ischemia in skeletal muscle: relationship to membrane potential, extracellular $\mathrm{pH}$, tissue lactic acid and ATP. Pflugers Arch. 404, 342-347.

Harashima, S., Horiuchi, T., Hatta, N., Morita, C. Higuchi, M., Sawabe, T., Tsukamoto, H., Tahira, T., Hayashi, K., Fujita, S., et al. (2001). Outside-toinside signal through the membrane TNF-alpha induces E-selectin (CD62E) expression on activated human CD4+ T cells. J. Immunol. 166 130-136.

Hirche, H., Franz, C., Bos, L., Bissig, R., Lang, R., and Schramm, M. (1980). Myocardial extracellular $\mathrm{K}+$ and $\mathrm{H}+$ increase and noradrenaline release as possible cause of early arrhythmias following acute coronary artery occlusion in pigs. J. Mol. Cell. Cardiol. 12, 579-593.

Holzer, P. (2009). Acid-sensitive ion channels and receptors. Handb. Exp. Pharmacol. 194, 283-332.

Huang, Y., and McNamara, J.O. (2004). Ischemic stroke: "acidotoxicity" is a perpetrator. Cell 118, 665-666.

Justus, C.R., and Yang, L.V. (2015). GPR4 decreases B16F10 melanoma cell spreading and regulates focal adhesion dynamics through the G13/Rho signaling pathway. Exp. Cell Res. 334, 100-113.
Justus, C.R., Dong, L., and Yang, L.V. (2013) Acidic tumor microenvironment and $\mathrm{pH}$-sensing G protein-coupled receptors. Front. Physiol. 4, 354.

Kozasa, T. Jiang, X. Hart, M.J. Sternweis, P.M. Singer, W.D., Gilman, A.G., Bollag, G., and Sternweis, P.C. (1998). p115 RhoGEF, a GTPase activating protein for Galpha12 and Galpha13. Science 280, 2109-2111.

Kumar, P., Shen, Q., Pivetti, C.D., Lee, E.S., Wu, M.H. and Yuan, S.Y. (2009). Molecular mechanisms of endothelial hyperpermeability: implications in inflammation. Expert Rev. Mol. Med. 11, e19.

Lardner, A. (2001). The effects of extracellular pH on immune function. J. Leukoc. Biol. 69, 522-530.

Liu, J.P., Nakakura, T., Tomura, H., Tobo, M., Mogi, C., Wang, J.Q., He, X.D., Takano, M., Damirin, A., Komachi, M., et al. (2010). Each one of certain histidine residues in G-protein-coupled receptor GPR4 is critical for extracellular protoninduced stimulation of multiple G-proteinsignaling pathways. Pharmacol. Res. 61, 499-505.

Ludwig, M.G., Vanek, M., Guerini, D., Gasser, J.A., Jones, C.E., Junker, U., Hofstetter, H., Wolf, R.M., and Seuwen, K. (2003). Proton-sensing Gprotein-coupled receptors. Nature 425, 93-98.

Ma, X.D., Hang, L.H., Shao, D.H., Shu, W.W., Hu, X.L., and Luo, H. (2017). TDAG8 activation attenuates cerebral ischaemia-reperfusion injury via Akt signalling in rats. Exp. Neurol. 293, 115-123.

Matsuzaki, S. Ishizuka, T., Yamada, H. Kamide, Y., Hisada, T., Ichimonji, I., Aoki, H., Yatomi, M. Komachi, M., Tsurumaki, H., et al. (2011).

Extracellular acidification induces connective tissue growth factor production through protonsensing receptor OGR1 in human airway smooth muscle cells. Biochem. Biophys. Res. Commun. 413, 499-503.

Miltz, W., Velcicky, J., Dawson, J., LittlewoodEvans, A., Ludwig, M.G., Seuwen, K., Feifel, R. Oberhauser, B., Meyer, A., Gabriel, D., et al. (2017). Design and synthesis of potent and orally active GPR4 antagonists with modulatory effects 
on nociception, inflammation, and angiogenesis. Bioorg. Med. Chem. 25, 4512-4525.

Muller, W.A. (2003). Leukocyte-endothelial-cell interactions in leukocyte transmigration and the inflammatory response. Trends Immunol. 24, 327-334.

Okajima, F. (2013). Regulation of inflammation by extracellular acidification and proton-sensing GPCRs. Cell. Signal. 25, 2263-2271.

Pate, M., Damarla, V., Chi, D.S., Negi, S., and Krishnaswamy, G. (2010). Endothelial cell biology: role in the inflammatory response. Adv. Clin. Chem. 52, 109-130.

Qiao, J., Huang, F., Naikawadi, R.P., Kim, K.S., Said, T., and Lum, H. (2006)

Lysophosphatidylcholine impairs endothelial barrier function through the $\mathrm{G}$ protein-coupled receptor GPR4. Am. J. Physiol. Lung Cell. Mol. Physiol. 291, L91-L101.

Rajamaki, K. Nordstrom, T. Nurmi, K. Akerman K.E., Kovanen, P.T., Oorni, K., and Eklund, K.K. (2013). Extracellular acidosis is a novel danger signal alerting innate immunity via the NLRP3 inflammasome. J. Biol. Chem. 288, 13410-13419.

Russell, J.L., Goetsch, S.C., Aguilar, H.R., Coe, H. Luo, X., Liu, N., van Rooij, E., Frantz, D.E., and Schneider, J.W. (2012). Regulated expression of $\mathrm{pH}$ sensing G Protein-coupled receptor-68 identified through chemical biology defines a new drug target for ischemic heart disease. ACS Chem. Biol. 7, 1077-1083.

Sanderlin, E.J., Justus, C.R. Krewson, E.A., and Yang, L.V. (2015). Emerging roles for the $\mathrm{pH}$ sensing $\mathrm{G}$ protein-coupled receptors in response to acidotic stress. Cell Health Cytoskelet. 7 99-109.
Sanderlin, E.J., Leffler, N.R., Lertpiriyapong, K. Cai, Q., Hong, H., Bakthavatchalu, V., Fox, J.G., Oswald, J.Z., Justus, C.R., Krewson, E.A., et al. (2017). GPR4 deficiency alleviates intestinal inflammation in a mouse model of acute experimental colitis. Biochim. Biophys. Acta 1863 569-584.

Sanderlin, E.J., Marie, M., Velcicky, J., Loetscher, P., and Yang, L.V. (2019). Pharmacological inhibition of GPR4 remediates intestinal inflammation in a mouse colitis model. Eur. J. Pharmacol. 852, 218-230.

Siesjo, B.K., Katsura, K.I., Kristian, T., Li, P.A., and Siesjo, P. (1996). Molecular mechanisms of acidosis-mediated damage. Acta Neurochir. Suppl. 66, 8-14.

Thennes, T. and Mehta, D. (2012). Heterotrimeric $\mathrm{G}$ proteins, focal adhesion kinase, and endothelial barrier function. Microvasc. Res. 83, $31-44$.

Tobo, A., Tobo, M., Nakakura, T., Ebara, M. Tomura, H., Mogi, C., Im, D.S., Murata, N. Kuwabara, A., Ito, S., et al. (2015). Characterization of imidazopyridine compounds as negative allosteric modulators of protonsensing GPR4 in extracellular acidificationinduced responses. PLoS One 10, e0129334.

Ulyanova, T., Scott, L.M., Priestley, G.V., Jiang, Y, Nakamoto, B., Koni, P.A., and Papayannopoulou, T. (2005). VCAM-1 expression in adult hematopoietic and nonhematopoietic cells is controlled by tissue-inductive signals and reflects their developmental origin. Blood 106, 86-94.

Vainer, B. Nielsen, O.H. and Horn, T. (1998). Expression of E-selectin, sialyl Lewis $\mathrm{X}$, and macrophage inflammatory protein-1alpha by colonic epithelial cells in ulcerative colitis. Dig. Dis. Sci. 43, 596-608. van Buul, J.D., and Hordijk, P.L. (2004). Signaling in leukocyte transendothelial migration. Arterioscler. Thromb. Vasc. Biol. 24, 824-833.

Velcicky, J., Miltz, W., Oberhauser, B., Orain, D. Vaupel, A., Weigand, K., Dawson King, J., Littlewood-Evans, A., Nash, M., Feifel, R., et al. (2017). Development of selective, orally active GPR4 antagonists with modulatory effects on nociception, inflammation, and angiogenesis. J. Med. Chem. 60, 3672-3683.

Wang, Y., de Valliere, C., Imenez Silva, P.H., Leonardi, I., Gruber, S., Gerstgrasser, A., Melhem, H., Weber, A., Leucht, K., Wolfram, L., et al. (2018) The proton-activated receptor GPR4 modulates intestinal inflammation. J. Crohns Colitis 12 355-368.

Wemmie, J.A., Price, M.P., and Welsh, M.J. (2006). Acid-sensing ion channels: advances, questions and therapeutic opportunities. Trends Neurosci. 29, 578-586.

Yang, L.V., Radu, C.G., Wang, L., Riedinger, M. and Witte, O.N. (2005). Gi-independent macrophage chemotaxis to ysophosphatidylcholine via the immunoregulatory GPCR G2A. Blood 105, 1127 1134.

Yang, L.V., Radu, C.G., Roy, M., Lee, S.

McLaughlin, J., Teitell, M.A., Iruela-Arispe, M.L. and Witte, O.N. (2007). Vascular abnormalities in mice deficient for the $\mathrm{G}$ protein-coupled receptor GPR4 that functions as a $\mathrm{pH}$ sensor. Mol. Cell. Biol. 27, 1334-1347.

Zhu, K., Baudhuin, L.M., Hong, G., Williams, F.S., Cristina, K.L., Kabarowski, J.H., Witte, O.N., and Xu, Y. (2001). Sphingosylphosphorylcholine and lysophosphatidylcholine are ligands for the G protein-coupled receptor GPR4. J. Biol. Chem. 276, 41325-41335, Retraction, 280, 43280, 2005. 
iScience, Volume 23

Supplemental Information

The Proton-Sensing GPR4 Receptor Regulates

Paracellular Gap Formation and Permeability

of Vascular Endothelial Cells

Elizabeth A. Krewson, Edward J. Sanderlin, Mona A. Marie, Shayan Nik Akhtar, Juraj Velcicky, Pius Loetscher, and Li V. Yang 


\section{Supplemental Figures}

A GPR4 Relative Expression

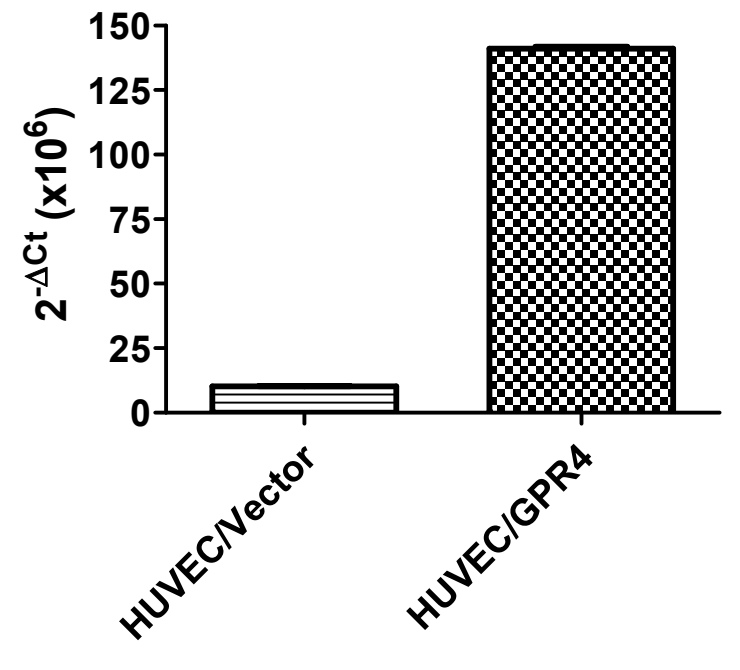

B GPR4 Relative Expression
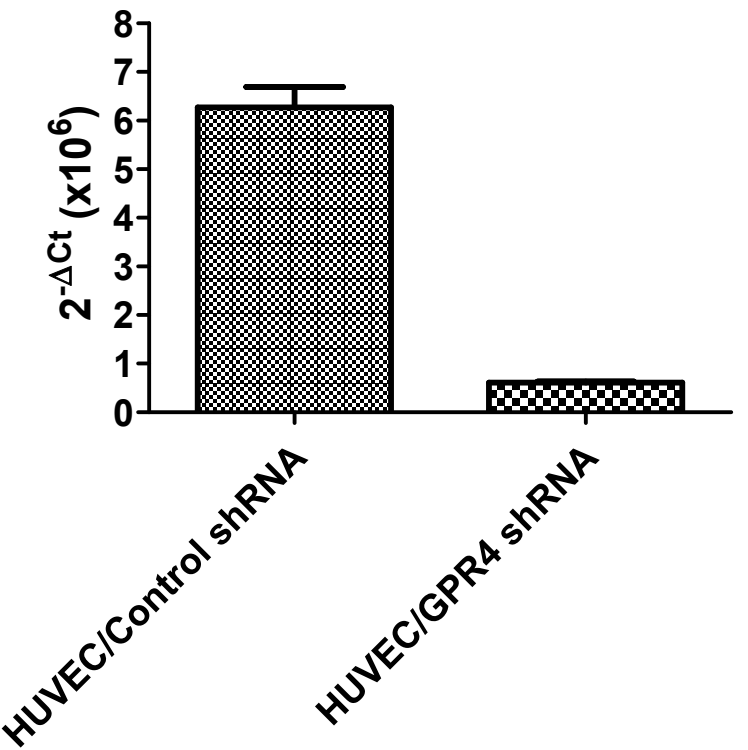

Figure S1. Expression of GPR4 mRNA in HUVECs. Related to Figure 2. (A and B) Real-time RTPCR was performed to assess the expression of GPR4 mRNA in HUVEC/Vector, HUVEC/GPR4, HUVEC/Control shRNA and HUVEC/GPR4 shRNA cells. GPR4 was overexpressed 14 fold in the HUVEC/GPR4 cells and knocked down $>90 \%$ in the HUVEC/GPR4 shRNA cells when compared to the control HUVEC cells (A and B, respectively). Each cDNA sample was run in duplicate for the real-time RT-PCR analysis, and the 18S rRNA was used as the internal control for data normalization. The $2^{-\Delta C t}$ method was used for data analysis. 

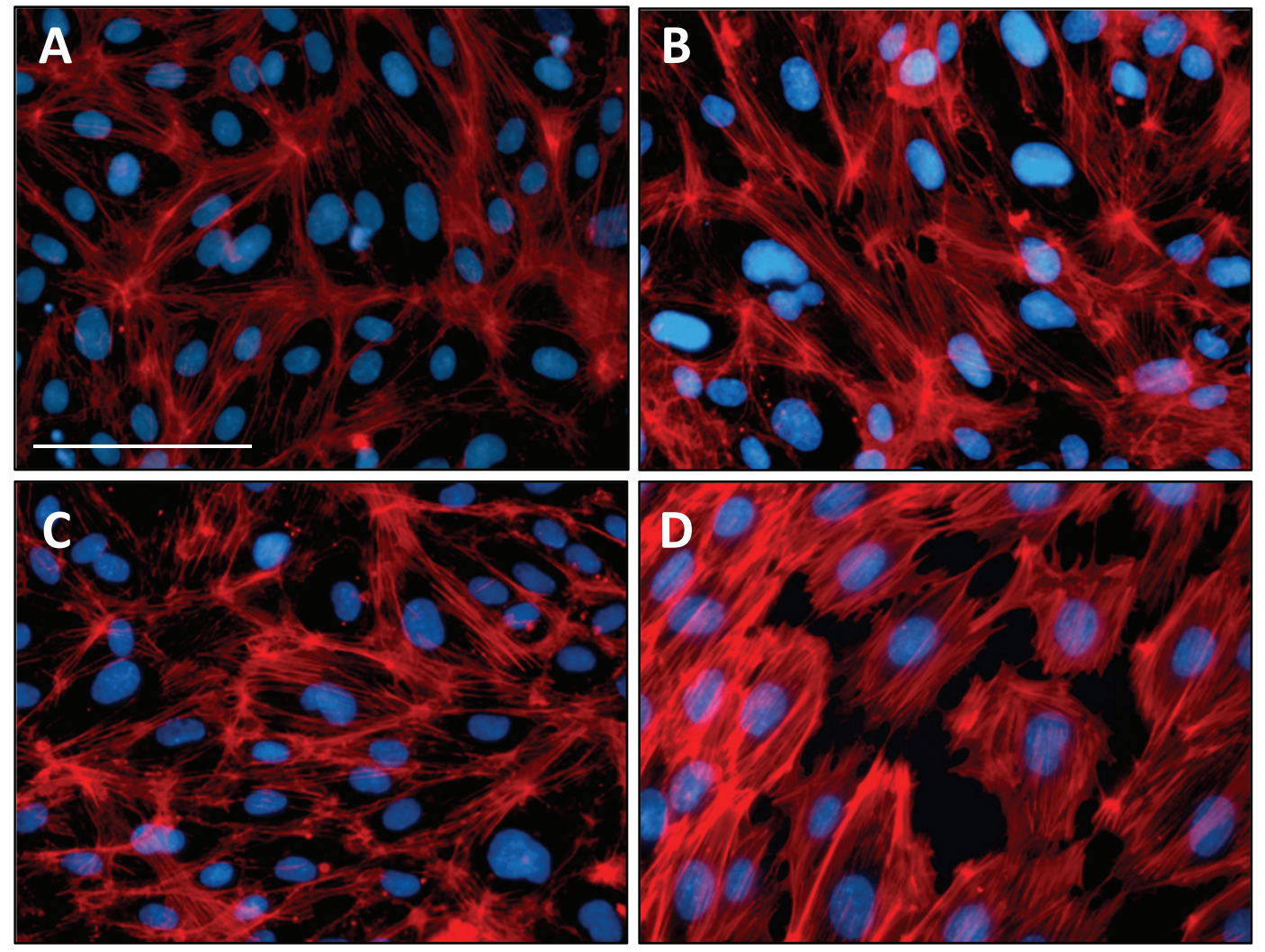

Figure S2. GPR4 activation by acidic pH induces actin stress fiber formation in HUVECs. Related to Figure 3. (A-D) Actin stress fiber formation in HUVECs treated with physiological or acidic pH. Activation of GPR4 by acidic pH 6.4 increases actin stress fibers when compared to physiological pH 7.4 in HUVECs. Representative images of F-actin labeled with rhodamine phalloidin in (A) HUVEC/vector $\mathrm{pH} 7.4$, (B) HUVEC/vector $\mathrm{pH} 6.4$, (C) HUVEC/GPR4 pH 7.4, and (D) HUVEC/GPR4 pH 6.4. HUVECs were treated with $\mathrm{pH}$ stimulation medium for 5 hours. Scale bar indicates $100 \mu \mathrm{m}$. 

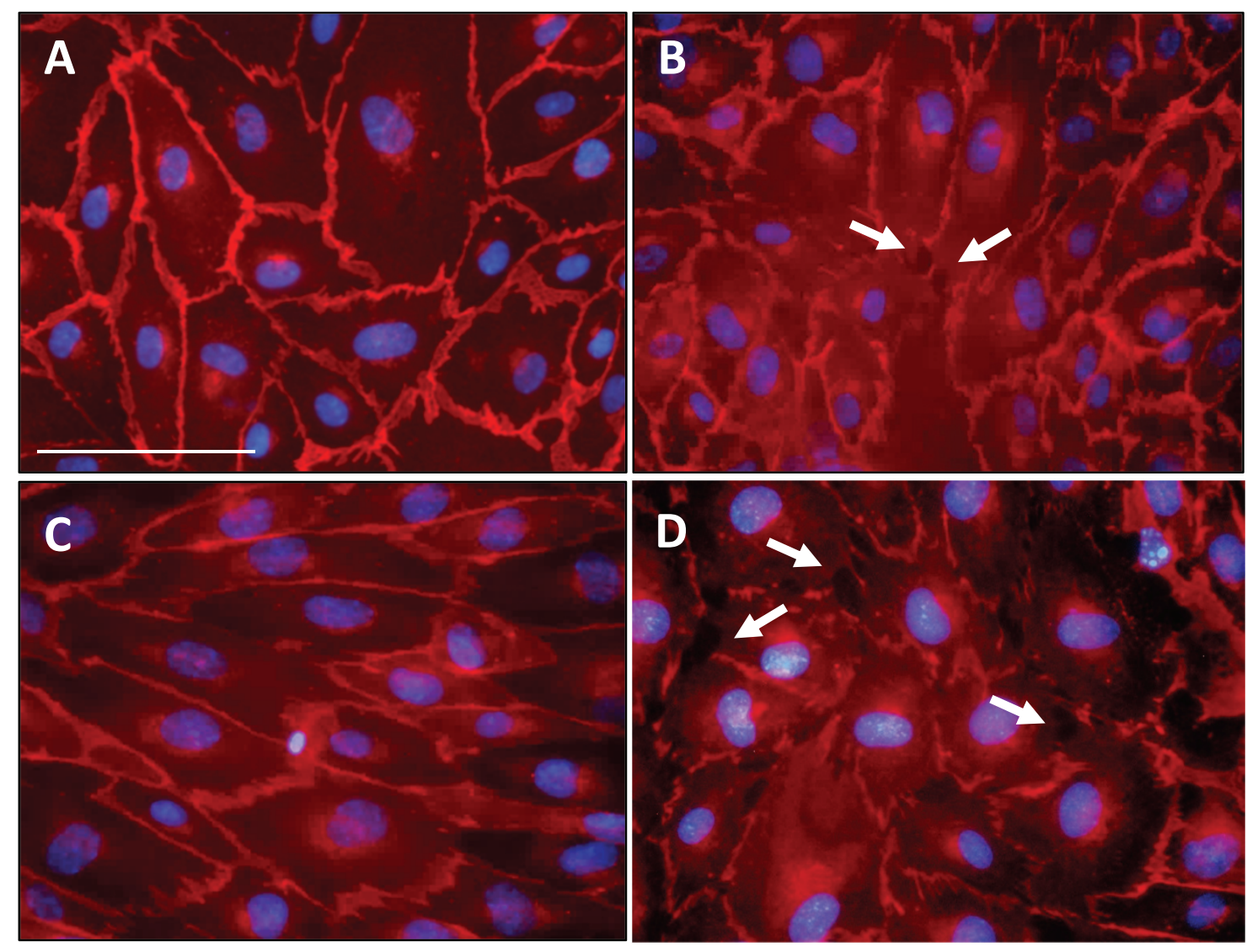

Figure S3. VE-Cadherin protein expression is reduced on endothelial paracellular gap borders. Related to Figure 3. (A-D) VE-Cadherin protein expression in HUVECs treated with physiological or acidic $\mathrm{pH}$. Acidosis/GPR4-mediated endothelial paracellular gap areas have reduced VECadherin expression along endothelial cell borders. Representative images of VE-Cadherin expression in (A) HUVEC/vector pH 7.4, (B) HUVEC/vector pH 6.4, (C) HUVEC/GPR4 pH 7.4, and (D) HUVEC/GPR4 pH 6.4. HUVECs were treated with $\mathrm{pH}$ stimulation medium for 5 hours. Scale bar indicates $100 \mu \mathrm{m}$. Arrows indicate endothelial cell borders with reduced VE-Cadherin protein expression. 

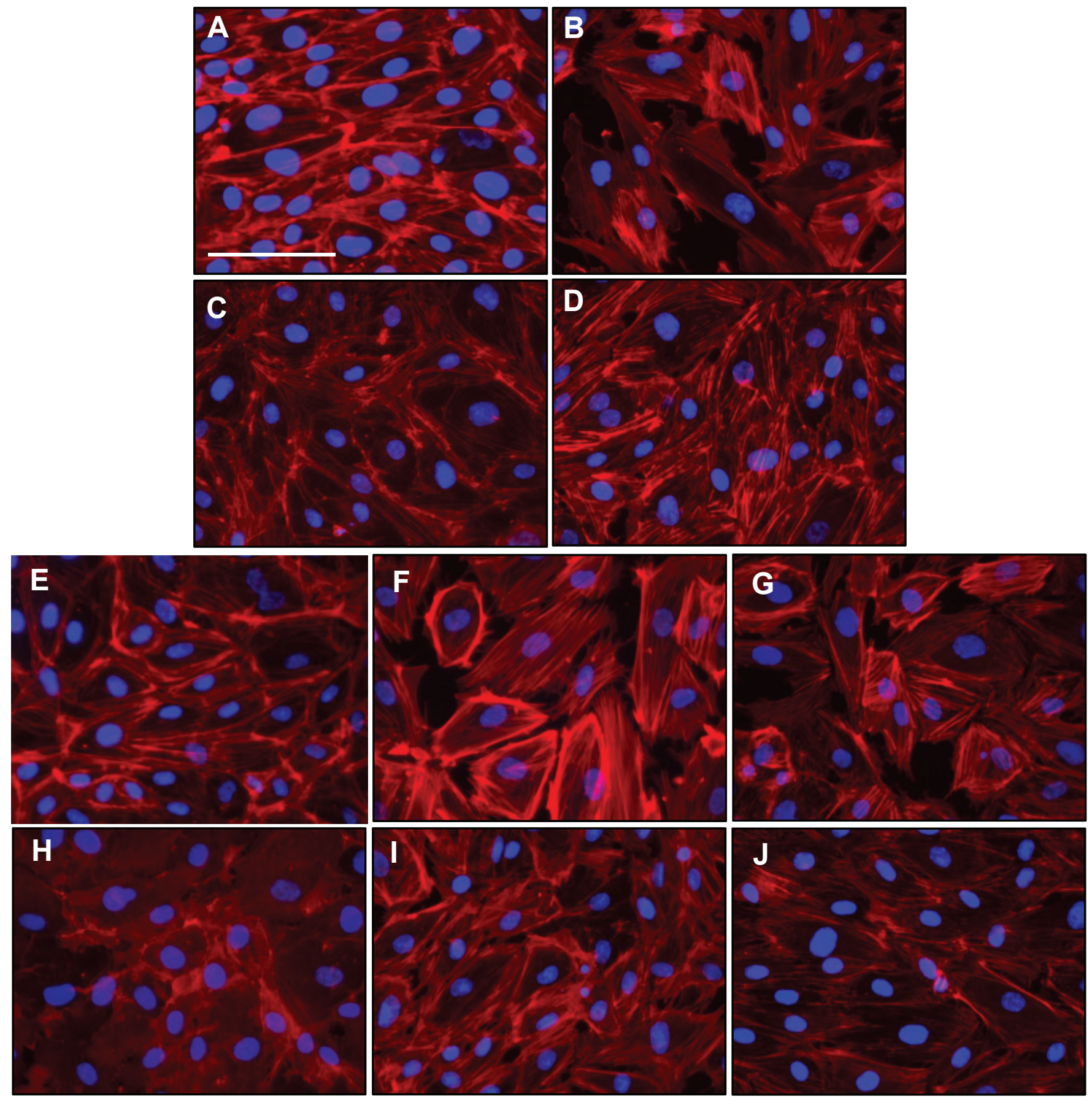

Figure S4. Inhibition of the $\mathrm{G}_{\alpha 12 / 13} /$ Rho GTPase/ROCK/MLCK/actin cytoskeleton pathway decreases acidosis/GPR4-induced actin stress fiber formation in HUVECs. Related to Figure 3. (A-J) Actin stress fiber formation in HUVEC/GPR4 cells treated with various inhibitors, including p115-RGS (an inhibitory construct of $\mathrm{G}_{\alpha 12 / 13}$ ), thiazovivin (a Rho-associated kinase ROCK inhibitor), staurosporine (a myosin light chain kinase MLCK inhibitor), and cytochalasin $\mathrm{D}$ (an actin cytoskeleton inhibitor). Representative images of F-actin labeled with rhodamine phalloidin in (A) HUVEC/GPR4/pQCXIP vector pH 7.4, (B) HUVEC/GPR4/pQCXIP vector pH 6.4, (C) HUVEC/GPR4/p115-RGS pH 7.4, (D) HUVEC/GPR4/p115-RGS pH 6.4, (E) HUVEC/GPR4 pH 7.4, (F) HUVEC/GPR4 pH 6.4, (G) HUVEC/GPR4 pH 6.4 DMSO, (H) HUVEC/GPR4 pH 6.4 thiazovivin, (I) HUVEC/GPR4 pH 6.4 staurosporine, and (J) HUVEC/GPR4 pH 6.4 cytochalasin D. HUVECs were treated with pH stimulation medium for 5 hours. Scale bar indicates $100 \mu \mathrm{m}$. 


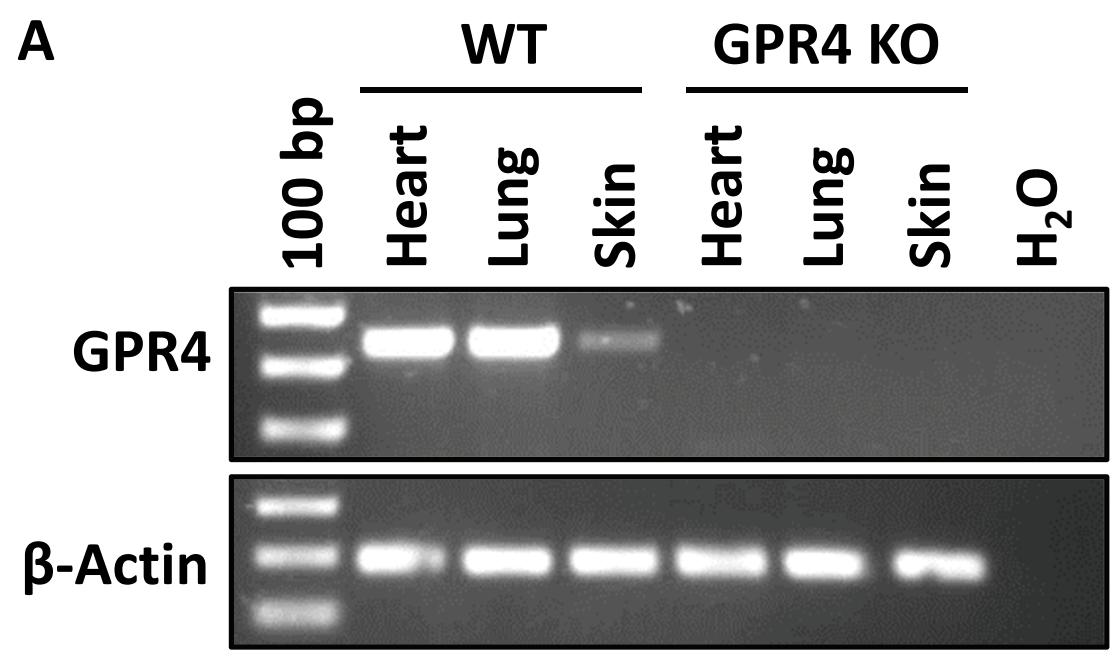

B

\section{GPR4 Relative Expression}

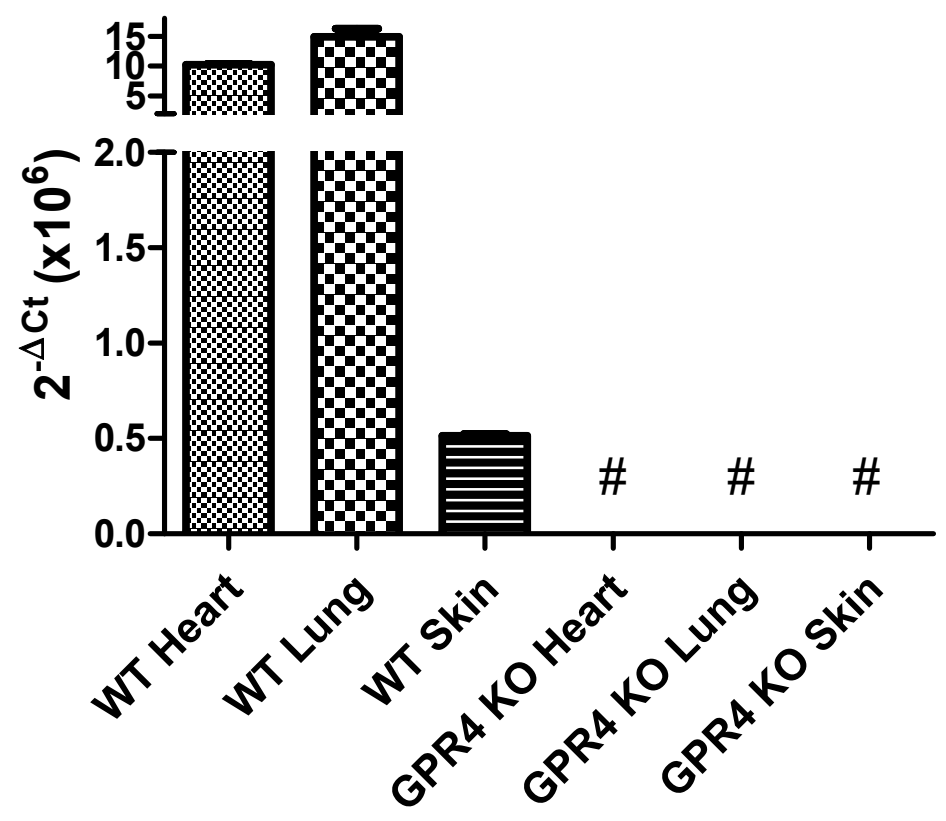

Figure S5. Absence of GPR4 mRNA expression in the GPR4 knockout mouse tissues. Related to Figures 5, 6 and 7. (A) RT-PCR and (B) real-time RT-PCR were performed to assess the expression of GPR4 mRNA in wild-type (WT) and GPR4 knockout (KO) mouse tissues. GPR4 mRNA expression was detected in the WT mouse heart, lung, and skin tissues but was absent in the GPR4 KO mouse tissues. Each cDNA sample was run in duplicate for the real-time RT-PCR analysis, and the $18 \mathrm{~S}$ rRNA was used as the internal control for data normalization. The $2^{-\Delta \mathrm{Ct}}$ method was used for data analysis. \# not detectable. 

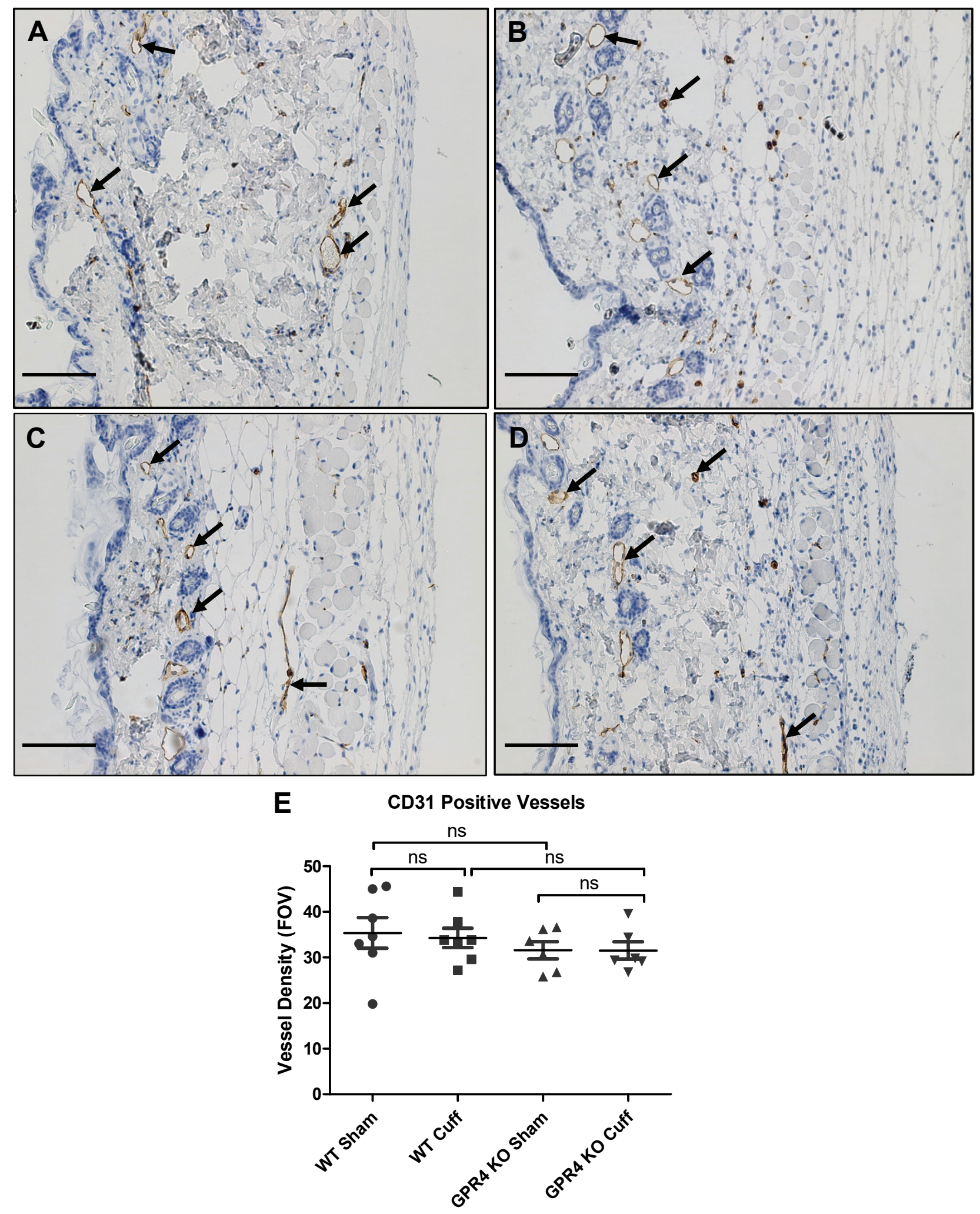

Figure S6. Blood vessel density in WT and GPR4 KO mouse hindlimb dermal tissues. Related to Figure 6. (A-E) Blood vessels were delineated by immunohistochemistry (IHC) with a pan-endothelial cell marker CD31 in the sham or cuff-affected mouse hindlimb dermal tissues. (A) WT sham, (B) WT cuff, (C) GPR4 KO sham, (D) GPR4 KO cuff, and (E) blood vessel density per field of view (FOV). No significant difference in blood vessel density was observed between WT and GPR4 KO or between sham and cuff affected dermal tissues. Arrows indicate blood vessels. 20x microscope objectives used. Scale bars indicate 100 $\mu \mathrm{m}$. Data are presented as mean \pm SEM and was analyzed for statistical significance using the ANOVA. ns: not significant. 


\section{Serum C-reactive protein}

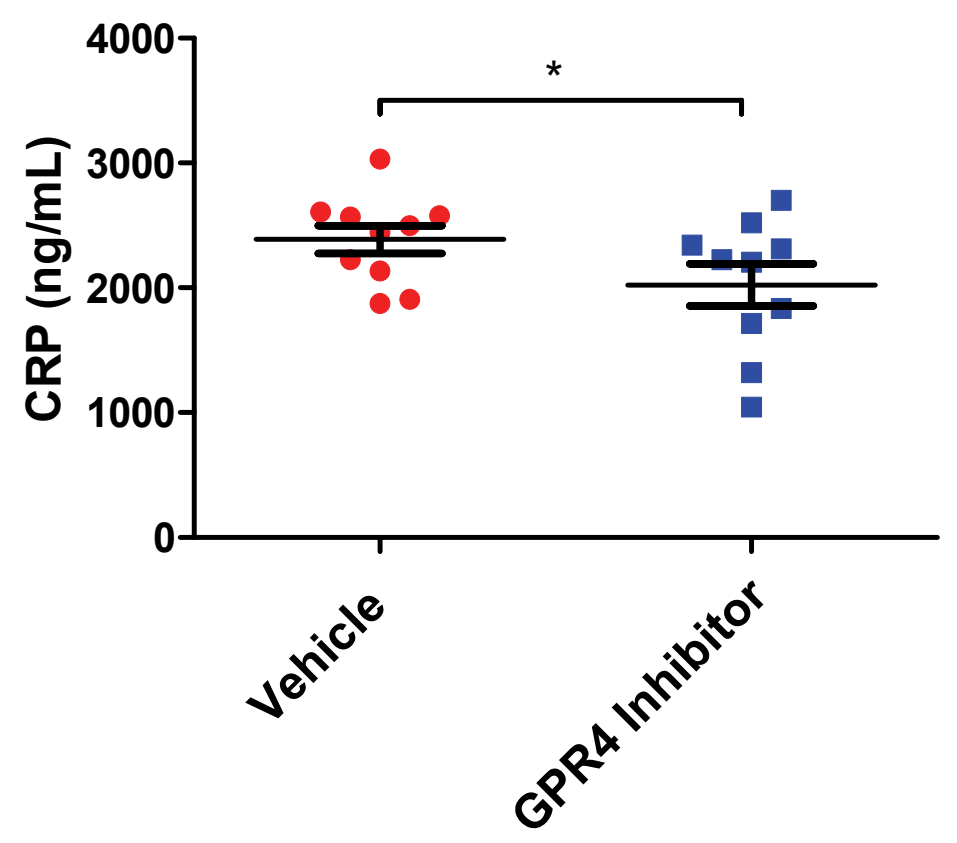

Figure S7. Mice treated with GPR4 antagonist 13 have reduced serum $\mathrm{C}$-reactive protein levels when compared to vehicle control. Related to Figure 9. C-reactive protein (CRP) inflammatory marker in mouse serum. As previous indicators suggest GPR4 antagonist 13 reduces inflammation in the hindlimb ischemia-reperfusion mouse model, we assessed the anti-inflammatory effects of GPR4 antagonist 13 in the reduction of CRP levels in mouse serum. Mouse serum CRP was measured by ELISA and represented as $\mathrm{ng} / \mathrm{mL}$. Data analyzed from $10 \mathrm{WT} /$ vehicle and $10 \mathrm{WT} / \mathrm{GPR} 4$ antagonist 13 mice. Data are presented as mean \pm SEM and analyzed for statistical significance using the unpaired one-tailed $t$-test where ${ }^{*} p<0.05$. 


\section{Transparent Methods}

Chemicals and reagents. For endothelial cell monolayer verification and live-cell staining, CellMask Deep Red Plasma Membrane Stain (\#C10046) and NucBlue Live ReadyProbes Reagent (\#R37605) were purchased from ThermoFisher Scientific (Waltham, MA, USA). The monoclonal antibody for VE-Cadherin (D87F2) was purchased from Cell Signaling Technology (Danvers, MA, USA). Secondary antibody, Rhodamine Red-X goat anti-rabbit (ThermoFisher Scientific) was used for all immunofluorescence experiments. Cellular permeabilization agent Triton X-100 detergent was purchased from BioRad (Hercules, CA, USA). Rhodamine Phalloidin used for Factin florescence staining was purchased from Cytoskeleton (Denver, CO, USA). For immunofluorescence staining, we used Vectashield Antifade Mounting Medium with DAPI from Vector Laboratories (Burlingame, CA, USA). To evaluate in vitro endothelial cell permeability, fluorescein isothiocyanate (FITC)-dextran (average molecular weight 10,000 Daltons) was purchased from Millipore Sigma (St. Louis, MO, USA). The chemicals used for buffering the $\mathrm{pH}$ stimulation media include 4-(2-hydroxyethyl)-1-piperazineethanesulfonic acid (HEPES), N-(2hydroxyethyl)-piperazine-N'-3-propanesulfonic acid (EPPS), 2-(4-morpholino)-ethanesulfonic acid (MES) and were purchased from Sigma-Aldrich (St Louis, MO, USA).

The GPR4 inhibitor, 2-Ethyl-3-(4-(E-3-(4-isopropyl-piperazin-1-yl)-propenyl)-benzyl)5,7-dimethyl-3H-imidazo(4,5-b)pyridine (abbreviated as EIDIP), was purchased from Dalton Pharma Services (Toronto, ON, Canada) as previously described (Dong et al., 2017; Dong et al., 2013). The new generation of GPR4 inhibitor, 2-(4-((2-Ethyl-5,7-dimethylpyrazolo[1,5a]pyrimidin-3-yl)methyl)phenyl)-5-(piperidin-4-yl)-1,3,4-oxadiazole (GPR4 antagonist 13, also known as NE-52-QQ57), was provided by Novartis Pharmaceuticals (Basel, Switzerland). The 
vehicle for GPR4 antagonist 13 was a combination of $0.5 \%$ methylcellulose purchased from BioConcept AG (Allschwil, Switzerland) and 0.5\% Tween-80 purchased from Sigma-Aldrich (St. Louis, MO, USA) and 99\% water.

Cell culture. Primary human umbilical vein endothelial cells (HUVEC), human pulmonary artery endothelial cells (HPAEC), and human lung microvascular endothelial cells (HMVEC-Lung) (Lonza, Walkersville, MD, USA), and primary human colon microvascular endothelial cells (HMVEC-Colon) (Cell Biologics Inc., Chicago, IL, USA) were cultured at $37^{\circ} \mathrm{C}$ and $5 \% \mathrm{CO}_{2}$ in a humidified incubator for experimentation. HUVEC and HPAEC were cultured in endothelial cell growth medium 2 (EGM-2), while HMVEC-Lung and HMVEC-Colon were grown in EGM-2MV medium (Lonza, Walkersville, MD, USA).

HUVECs stably expressing MSCV-IRES-GFP (HUVEC/Vector), MSCV-huGPR4-IRESGFP (HUVEC/GPR4), MSCV-huGPR4 R115A-IRES-GFP (HUVEC/GPR4-R115A), Flinkcontrol shRNA (HUVEC/Control shRNA), or Flink-huGPR4 shRNA (HUVEC/GPR4 shRNA) were developed by retroviral transduction and sorted by fluorescence-activated cell sorting (FACS) as previously described (Chen et al., 2011; Dong et al., 2017; Dong et al., 2013). To generate $\mathrm{G}_{12 / 13}$ signaling deficient HUVECs, the p115 RGS construct was cloned into the pQCXIP vector as previously described (Kozasa et al., 1998; Yang et al., 2005). The p115 RGSpQCXIP and empty pQCXIP retroviral vectors were stably transduced into the HUVEC/vector and HUVEC/GPR4 cells which were selected with $2 \mu \mathrm{g} / \mathrm{mL}$ puromycin for 48 hours. Cells were used within 8 passages for all experiments. 
Gap formation assay. Endothelial cells were seeded at $1 \times 10^{5}$ cells/well in a 24 -well tissue culture treated plate and were cultured to a confluent monolayer. Intact cellular monolayers were confirmed using a deep red cellular membrane dye (ThermoFisher, \#C10046, Waltham, MA, USA) and a live cell nuclear dye (ThermoFisher, \#R37605, Waltham, MA, USA). Endothelial cells were then treated with cell culture medium buffered to physiological pH 7.4 or acidic $\mathrm{pH} 6.4$ for defined time points as previously described (Chen et al., 2011; Dong et al., 2017; Dong et al., 2013). For assessment of pharmacological inhibition of GPR4 in vitro, the GPR4 inhibitor (EIDIP, Dalton Pharma) was added to the $\mathrm{pH}$ stimulation media following the GPR4 inhibitor pretreatment for 1 hour in physiological pH 7.4 (Dong et al., 2017; Dong et al., 2013). Pictures of endothelial cell monolayers were obtained hourly over a 5-hour period using the EVOS Digital Inverted Microscope imaging system. ImageJ software (NIH) was used to quantify gap percentage per field of view (FOV).

Fluorescent filamentous-actin stress fiber formation assessment. Following the gap formation assay, endothelial cells were washed with DPBS and fixed with 4\% paraformaldehyde for 15 minutes. Cells were then permeabilized for 10 minutes with $0.1 \%$ Triton X-100 and stained with Rhodamine Phalloidin for 30 minutes (Cytoskeleton Inc., Denver, CO, USA). Cells were then washed in DPBS and mounted with DAPI-containing Vectashield mounting media (Vector Laboratories, H-1200, Burlingame, CA, USA). Cells were imaged using the EVOS Digital Inverted Microscope imaging system.

Immunofluorescence. The endothelial cell monolayers in 24-well plates were treated with physiological $\mathrm{pH} 7.4$ or acidic $\mathrm{pH} 6.4$ media for 5 hours. Following pH treatments, cells were fixed 
with $4 \%$ paraformaldehyde for 15 minutes, permeabilized with $0.1 \%$ Triton X-100 for 5 minutes and blocked with goat serum in phosphate buffered saline with $0.1 \%$ Tween-20 for 1 hour. The cells were then incubated with VE-cadherin primary antibody (Cell Signaling Technology, D87F2) in the blocking solution overnight at $4^{\circ} \mathrm{C}$. Cells were washed and incubated for 1 hour with Rhodamine Red-X goat anti-rabbit secondary antibody. The wells were then washed five times with PBS and mounted with a glass cover slip using Vectashield with DAPI. The stained cells were viewed and pictures taken with an EVOS Digital Inverted Microscope.

Cellular permeability assay. Endothelial cell monolayers were cultured to confluency on a Transwell membrane insert with $0.4 \mu \mathrm{m}$ pores (Corning, NY, USA) within a 24 -well plate and subsequently treated with physiological $\mathrm{pH} 7.4$ or acidic $\mathrm{pH} 6.4$ media for 5 hours. Following $\mathrm{pH}$ treatment, fluorescein isothiocyanate (FITC)-dextran with average molecular weight of 10,000 daltons (Millipore Sigma, St. Louis, MO, USA) diluted into pH stimulation medium was added into the upper chamber of the Transwell insert at a concentration of $1 \mathrm{mg} / \mathrm{mL}$. Subsequently, $1 \mathrm{~mL}$ of endothelial growth medium was also added to the lower chamber and the Transwell was incubated for 30 minutes at $5 \% \mathrm{CO}_{2}$ at $37^{\circ} \mathrm{C}$. Following the incubation, $100 \mu \mathrm{L}$ of medium was drawn from the lower chamber and the FITC-dextran signal was quantified with a microplate reader at excitation/emission wavelength of $490 / 520 \mathrm{~nm}$. The degree of cell permeability was determined by comparing the FITC-dextran fluorescent signal from the treatment group to the control group.

Tourniquet-based acute hindlimb ischemia and reperfusion murine model. All animal experiments were performed on 9-13-week old male and female mice. GPR4 wild-type (WT) or 
GPR4-deficient (GPR4 KO) mice backcrossed into the C57BL/6 genetic background for 11 generations were used (Yang et al., 2007). To assess the inflammatory response post ischemiareperfusion, the mouse hindlimb ischemia reperfusion injury model was employed using the pneumatic digital tourniquet cuff system (Model DC1.6, Hokanson, Inc., Bellevue, WA, USA). Prior to tourniquet application, a single dose of $0.03 \mathrm{mg} / \mathrm{kg}$ Buprenorphine analgesics was administered subcutaneously to each mouse. Mice were then anesthetized with $1 \%$ isoflurane inhalation and placed on a temperature-controlled pad followed by the tourniquet application to the left proximal hindlimb under $200 \mathrm{~mm} \mathrm{Hg}$ regulated pressure for $3 \mathrm{hrs}$ as previously described (Bonheur et al., 2004). During the tourniquet-induced hindlimb ischemia procedure, the mouse breathing rate and body temperature was monitored. Mice were injected subcutaneously with 0.5 $\mathrm{mL}$ warm saline at the 1.5 hour and 3 hour time points for maintenance of hydration. After the tourniquet was released, the mice were placed into the microisolation cages with soft bedding for a 21 hour hindlimb reperfusion. Hindlimb circumference was measured before the procedure (baseline) and after the ischemia-reperfusion (IR), and the change in hindlimb circumference (value after IR - value at baseline) was used as an indicator to assess tissue edema. For the evaluation of GPR4 antagonist 13 (also known as NE-52-QQ57, Novartis) in the ischemiareperfusion mouse model, both C57BL/6J and GPR4 WT mice were used for experiments ranging from 9 to 13 weeks old. Mice were administered vehicle control ( $0.5 \%$ methylcellulose/ $0.5 \%$ Tween $80 / 99 \%$ water) or GPR4 antagonist 13 at $30 \mathrm{mg} / \mathrm{kg}$ b.i.d. by oral gavage one day prior to experimental procedure. Mice were given a second dose of vehicle or GPR4 antagonist 13 approximately 2 hours prior to tourniquet cuff procedure. The last dose of either vehicle or GPR4 antagonist 13 was administered 6 hours into the reperfusion phase. The dosage of $30 \mathrm{mg} / \mathrm{kg}$ b.i.d. was chosen because of the best efficacy observed in a previous study (Velcicky et al., 2017). All 
animal experiments were approved by the Institutional Animal Care \& Use Committee of East Carolina University and were in accordance with the Guide for the Care and Use of Laboratory Animals administered by the Office of Laboratory Animal Welfare, NIH.

Tissue collection and histological analysis. After the 24 hour hindlimb ischemia-reperfusion procedure, mice were euthanized and the lower body was dissected for tissue collection. Upon dissection, inflammatory exudate within the interstitial space between upper leg muscle fibrous sheath and skin from the tourniquet-affected hindlimb was weighed and collected. A portion of the hindlimb skin containing inflammatory exudate was removed approximately $20 \times 6 \mathrm{~mm}$ and fixed in $10 \%$ buffered formalin for tissue processing. The remaining exudate on the skin of the mouse was dissected and weighed. Tissues were embedded in paraffin, sectioned at $7 \mu \mathrm{m}$, and stained with hematoxylin and eosin (H\&E) for histopathological analyses. For assessment of neutrophilic infiltration into inflammatory exudate, pictures were taken from $\mathrm{H} \& \mathrm{E}$ tissue sections at five random points with a 20x objective. Leukocytes with the distinct polymorphic nuclear morphology were counted in each field of view (FOV) using ImageJ software in a blind manner as previously described (Sanderlin et al., 2017). Pictures were taken using the Zeiss Axio Imager A1 microscope.

Immunohistochemistry. Immunohistochemistry (IHC) was performed on serial sections of $7 \mu \mathrm{m}$ paraffin embedded skin with inflammatory exudate. Tissues were deparaffinized and hydrated to water by a series of declining ethanol concentrations. Antigen retrieval was performed using TrisEDTA (pH 9.0) with 0.1\% Tween 20 to assess protein expression using the SuperPicture 3rd Gen Immunohistochemistry detection system (Invitrogen, Waltham, MA). Endogenous peroxidases 
were quenched and endogenous mouse IgG in blood serum was blocked using the Mouse-onMouse blocking reagent (Vector Laboratories, Burlingame, CA). Subsequently, 10\% normal goat serum was used to block tissue followed by primary antibody incubation with anti-VCAM-1 (Abcam, ab134047), E-selectin (Abcam, ab18981, Cambridge, MA), and CD31 (Cell Signaling Technology, \#77699, Danvers, MA) overnight at $4{ }^{\circ} \mathrm{C}$ and followed by incubation with secondary antibody. For detection, the Rabbit Vectastain Elite ABC-HRP Kit (Vector Laboratories, Burlingame, CA, USA) was employed according to the manufacture's protocol. After IHC was performed, pictures were taken using a Zeiss Axio Imager A1 microscope. VCAM-1 and Eselectin intensity in vascular endothelial cells were assessed using the scoring criteria as previously described: 1 = none/minimal, 2 = mild, 3 = moderate, 4 = high signal intensity (Sanderlin et al., 2019). To quantify blood vessel density, CD31-positive blood vessels per field of view (FOV) was counted.

To assess mouse plasma IgG protein in the exudate, the mouse Vectastain Elite ABC-HRP Kit was used (Vector Laboratories, Burlingame, CA). Endogenous peroxidase was quenched, blocking serum and biotinylated anti-mouse $\operatorname{IgG}$ secondary antibody was used followed by ImmPACT DAB Peroxidase Substrate (Vector Laboratories, Burlingame, CA) to visualize IgG leakiness from plasma. Immunohistochemical staining of tissue sections with no anti-mouse IgG antibody and the use of anti-Rabbit IgG antibody (Vector Laboratories, Burlingame, CA) were used for negative control. Pictures were taken with a Zeiss Axio Imager A1 microscope.

Enzyme-linked immunosorbent assay (ELISA). ELISA was performed to measure the Creactive protein (CRP) level in the serum of mice treated with the GPR4 antagonist 13 or vehicle control. Immediately after the mice were euthanized, blood was collected through cardiac puncture 
and allowed to form clots. After centrifugation, mouse serum on top of the clots was collected and stored in a $-80{ }^{\circ} \mathrm{C}$ freezer for further analysis. The CRP concentration in mouse serum was measured using the mouse CRP ELISA kit (Sigma-Aldrich, RAB1121) according to the manufacturer's protocol.

RNA isolation and real-time RT-PCR. Total RNA was isolated from HUVECs and mouse tissues using the DNA/RNA/Protein extraction kit (IBI Scientific, Peosta, IA) and reverse transcribed into cDNA using the SuperScript IV reverse transcriptase (ThermoFisher Scientific, Waltham, MA). Real-time PCR was performed in duplicate with a program of $50^{\circ} \mathrm{C}$ for $2 \mathrm{~min}$, $95^{\circ} \mathrm{C}$ for $10 \mathrm{~min}$ followed by 40 cycles of $95^{\circ} \mathrm{C}$ for $15 \mathrm{sec}$ and $60^{\circ} \mathrm{C}$ for $1 \mathrm{~min}$. Data was acquired using the QuantStudio 3 Real-Time PCR system (ThermoFisher Scientific, Waltham, MA) and analyzed using the $2^{-\Delta \mathrm{Ct}}$ method. TaqMan primer-probe sets used for gene expression analysis include 18S rRNA (Hs99999901_s1), mouse Gpr4 (Mm00558777_s1), and human GPR4 (custom designed) as previously described (Chen et al., 2011; Dong et al., 2013). Additionally, regular RTPCR was performed to assess mouse Gpr4 expression in WT and GPR4 KO mouse tissues as previously described (Yang et al., 2007). The mouse Gpr4 primer sequences for regular RT-PCR were: (sense) 5'-CAAGACCCACTTGGACCACA-3' and (antisense) 5'TGTCCTGGGCCTCCTTTCTA-3’

Statistical Analysis. GraphPad Prism software was used for statistical analysis. The results were recorded as the mean \pm standard error from at least three independent experiments. For three or more groups, ANOVA was used followed by Bonferroni post hoc test. Statistical analyses between two groups were performed using the $t$-test. $P<0.05$ was considered statistically significant. 


\section{Supplemental References}

Bonheur, J.A., Albadawi, H., Patton, G.M., and Watkins, M.T. (2004). A noninvasive murine model of hind limb ischemia-reperfusion injury. J Surg Res 116, 55-63.

Chen, A., Dong, L., Leffler, N.R., Asch, A.S., Witte, O.N., and Yang, L.V. (2011). Activation of GPR4 by acidosis increases endothelial cell adhesion through the cAMP/Epac pathway. PLoS One 6, e27586.

Dong, L., Krewson, E.A., and Yang, L.V. (2017). Acidosis Activates Endoplasmic Reticulum Stress Pathways through GPR4 in Human Vascular Endothelial Cells. Int J Mol Sci 18.

Dong, L., Li, Z., Leffler, N.R., Asch, A.S., Chi, J.T., and Yang, L.V. (2013). Acidosis Activation of the Proton-Sensing GPR4 Receptor Stimulates Vascular Endothelial Cell Inflammatory Responses Revealed by Transcriptome Analysis. PLoS One 8, e61991.

Kozasa, T., Jiang, X., Hart, M.J., Sternweis, P.M., Singer, W.D., Gilman, A.G., Bollag, G., and Sternweis, P.C. (1998). p115 RhoGEF, a GTPase activating protein for Galpha12 and Galpha13. Science 280, 2109-2111.

Sanderlin, E.J., Leffler, N.R., Lertpiriyapong, K., Cai, Q., Hong, H., Bakthavatchalu, V., Fox, J.G., Oswald, J.Z., Justus, C.R., Krewson, E.A., et al. (2017). GPR4 deficiency alleviates intestinal inflammation in a mouse model of acute experimental colitis. Biochim Biophys Acta 1863, 569584.

Sanderlin, E.J., Marie, M., Velcicky, J., Loetscher, P., and Yang, L.V. (2019). Pharmacological inhibition of GPR4 remediates intestinal inflammation in a mouse colitis model. Eur J Pharmacol $852,218-230$.

Velcicky, J., Miltz, W., Oberhauser, B., Orain, D., Vaupel, A., Weigand, K., Dawson King, J., Littlewood-Evans, A., Nash, M., Feifel, R., et al. (2017). Development of Selective, Orally Active GPR4 Antagonists with Modulatory Effects on Nociception, Inflammation, and Angiogenesis. J Med Chem 60, 3672-3683.

Yang, L.V., Radu, C.G., Roy, M., Lee, S., McLaughlin, J., Teitell, M.A., Iruela-Arispe, M.L., and Witte, O.N. (2007). Vascular abnormalities in mice deficient for the G protein-coupled receptor GPR4 that functions as a pH sensor. Mol Cell Biol 27, 1334-1347.

Yang, L.V., Radu, C.G., Wang, L., Riedinger, M., and Witte, O.N. (2005). Gi-independent macrophage chemotaxis to lysophosphatidylcholine via the immunoregulatory GPCR G2A. Blood $105,1127-1134$. 